\title{
Imprecise random field analysis for non-linear concrete damage analysis
}

\author{
Mona M. Dannert ${ }^{a, 1}$, Matthias Faes ${ }^{b}$, Rodolfo M.N. Fleury ${ }^{a}$, Amelie Fau ${ }^{c}$, \\ Udo Nackenhorst $^{a}$, David Moens ${ }^{b}$ \\ ${ }^{a}$ Leibniz University Hannover, Institute of Mechanics and Computational Mechanics, Germany \\ ${ }^{b}$ KU Leuven, Department of Mechanical Engineering, Technology campus De Nayer, St.-Katelijne-Waver, Belgium \\ ${ }^{c}$ Université Paris-Saclay, ENS Paris-Saclay, CNRS, LMT-Laboratoire de Mécanique et Technologie, France
}

\begin{abstract}
Imprecise random fields consider both, aleatory and epistemic uncertainties. In this paper, spatially varying material parameters representing the constitutive parameters of a damage model for concrete are defined as imprecise random fields by assuming an interval valued correlation length. For each correlation length value, the corresponding random field is discretized by Karhunen-Loève expansion. In a first study, the effect of the series truncation is discussed as well as the resulting variance error on the probability box (p-box) that represents uncertainty on the damage in a concrete beam as a result of the imprecise random field. It is shown that a certain awareness for the influence of the truncation order on the local field variance is needed when the series is truncated according to a fixed mean variance error.

In the following case study, the main investigation is on the propagation of imprecise random fields in the context of non-linear finite element problems, i.e. quasi-brittle damage of a four-point bended concrete beam. The global and local damage as the quantities of interest are described by a p-box. The influence of several imprecise random field input parameters to the resulting p-boxes is studied. Furthermore, it is examined whether correlation length values located within the interval, so-called intermediate values, affect the p-box bounds. It is shown that, from the engineering point of view, a pure vertex analysis of the correlation length intervals is sufficient to determine the p-box in this context.
\end{abstract}

Keywords: Uncertainty quantification, imprecise random fields, interval valued correlation length, Karhunen-Loève expansion, non-linear stochastic finite element method, probability box approach

\section{Introduction}

Uncertainties are unavoidable in engineering practice, e.g. in terms of material, geometry or loading parameters. In conventional design, uncertainties are usually considered by safety or knock-down factors, however this may result in conservative and therefore more expensive designs. To enable resource-conserving

\footnotetext{
${ }^{1}$ mona.dannert@ibnm.uni-hannover.de
} 
5 but reliable designs, high research efforts are made to investigate sophisticated approaches including the required uncertainty quantification.

The first step to quantify uncertainties is to consider different origins. Distinction is typically made between aleatory (stochastic) and epistemic uncertainties [1]. While the first are due to the natural randomness of a phenomenon and can be handled by probabilistic methods, the latter are caused by a lack of knowledge or data. Therefore, stochastic parameters cannot be reliably quantified and possibilistic approaches such as interval [2] or fuzzy 3] theory are required. However, in reality uncertainties are usually neither purely aleatory nor purely epistemic but of mixed nature [4, 5], which is also referred to as polymorphic or deep uncertainties by some authors [6, 7]. Those mixed uncertain input parameters demand for appropriate approaches, as e.g. evidence theory [8], fuzzy probability theory [9] or probability bounds analysis [10]. Over the last years, several highly efficient approaches to propagate imprecise probabilities have been introduced in literature. For instance, in reference [11, a linear programming method is presented to compute bounds on the probability of failure of structures subjected to imprecise probabilistic uncertainty. Other approaches include the application of variants of the Sobol-Hoeffding decomposition (also known as high dimensional model representation or HDMR) in combination with extended Monte Carlo simulation [12] or line sampling [13. In reference [14, a method is introduced to propagate imprecise probabilities by combining Improved Interval Analysis via Extra Unitary Interval in combination with classical probabilistic analysis. Also several surrogate modelling schemes for the propagation of imprecise probabilities have been introduced in literature, based on e.g., polynomial chaos expansions [15] or Interval Predictor Models [16].

Within this paper, mixed aleatory and epistemic uncertain material properties are considered in the context of non-linear finite element models. The non-linearity of the problem results from a brittle damage law. As material properties can vary within a component it is often reasonable to describe them as random fields. This means that they vary not only on chance from one sample to the next but also spatially within the sample itself. Random fields are classically aleatory uncertainties and described by a Karhunen-Loève expansion [17 based on a mean value and standard deviation of the field as well as a certain correlation structure. For many usual correlation functions the correlation length describes the correlation of two arbitrary values within the parameter field. Usually, mean value and standard deviation can be determined by experiments, while the correlation length is difficult to determine experimentally. However, as it impacts both the modelled variance of the field as well as its numerical discretization, the correlation length is as such a crucial factor that governs the modelling and simulation of the random field [18. For these reasons, it is proposed to be described by an interval in this paper to acknowledge any epistemic uncertainty that is present in its definition.

This special mixed uncertain properties are then called imprecise random fields and have been investigated in several settings. In terms of material parameters, the mean value and standard deviation are considered interval valued in [19], while interval and fuzzy valued correlation lengths are explored by [20]. 
Recent applications of imprecise random fields were presented in [7] for geometric imperfections and in [21] to propagate imprecise stochastic loads through linear dynamical models.

The imprecise random field material parameters are propagated by a probability box approach in this paper. Here, the probability is not defined by a certain value but by an upper and lower bound called probability box (p-box). A straightforward approach is to discretize the epistemic uncertainties 22]. However, for non-linear finite element (FE) problems, this can become computational costly and it cannot be guaranteed that all crucial values within the interval are considered. In reference [23] a mathematical framework for the description and propagation of imprecise random fields is provided. The authors also provide an optimization approach to find the crucial values within the interval a-priori and studied the effect of these so-called intermediate values. However, application of this concept is scarce in literature and the impact of intermediate values in general engineering practice is as yet unclear. This paper therefore aims at investigating the influence of imprecise random field input parameters within concrete damage simulation. Such highly non-linear FE analyses increase the computational cost of an individual realization notably. Considering additional intermediate values besides a pure vertex analysis therefore can increase the cost of an imprecise random field propagation significantly, especially when several input parameters are regarded as imprecise random fields. For this reason, beside the influence of intermediate values, especially the sensitivity of several imprecise random field input parameters is studied. The question whether and how these interfere with each other is inevitable to estimate the influence on the resulting p-box when making parameter assumptions. For this purpose, the damage evolution of a four-point bending concrete beam is investigated considering one and two material parameters as imprecise random fields.

This paper is structured as follows: Section 2 reviews two concepts to model spatial uncertainties, random fields for aleatory uncertainties and interval fields for epistemic uncertainties. Afterwards, a hybrid approach is introduced to model both kinds of uncertainties mixed in terms of imprecise random fields in Section 3 To apply this approach to a damage mechanics problem, the meaning of the underlying material model and properties as well as the challenges in describing these by Karhunen-Loève expansion are discussed in Section 4. Based on that, the influence of imprecise random fields on different quantities of interest of such a non-linear finite element problem is investigated in Section 5 .

\section{Modelling concepts for spatial uncertainty analysis}

In terms of uncertainty quantification, often a distinction between two kinds of uncertainties is made based on their respective source: those parameters that originate from the intrinsic randomness of a phenomenon itself are classified as aleatory uncertain while those that follow from a lack of knowledge or data are called epistemic uncertain [1]. Uncertain parameters can furthermore depend on space or time. The 
modelling of these kinds of uncertainties requires special attention in terms of defining their autocorrelation structure.

In this section, two strategies to deal with spatially uncertain properties are discussed: random fields to

a Karhunen-Loève (KL) series expansion [31]

$$
x(\boldsymbol{z}, \theta)=\mu_{x}(\boldsymbol{z})+\sigma_{x} \sum_{i=1}^{\infty} \sqrt{\lambda_{i}} \boldsymbol{\psi}_{i}(\boldsymbol{z}) \xi_{i}(\theta),
$$

with $\xi_{i}(\theta), i=1, \ldots, \infty$ uncorrelated random variables, which are determined according to:

$$
\xi_{i}(\theta)=\frac{1}{\sqrt{\lambda_{i}}} \int_{\Omega}\left[x(\boldsymbol{z}, \theta)-\mu_{x}(\boldsymbol{z})\right] \boldsymbol{\psi}_{i}(\boldsymbol{z}) \mathrm{d} \boldsymbol{z},
$$


which can be shown to be independent standard normally distributed in the case of a Gaussian random field. The quantities $\lambda_{i} \in(0, \infty)$ and $\boldsymbol{\psi}_{i}(\boldsymbol{z}): \Omega \mapsto \mathbb{R}$ are respectively the eigenvalues and eigenfunctions of the continuous, bounded, symmetric and positive (semi-)definite autocorrelation function $\boldsymbol{\Gamma}_{x}\left(\boldsymbol{z}, \boldsymbol{z}^{\prime}\right)$, in accordance with Mercer's theorem:

$$
\boldsymbol{\Gamma}_{x}\left(\boldsymbol{z}, \boldsymbol{z}^{\prime}\right)=\sum_{i=1}^{\infty} \lambda_{i} \boldsymbol{\psi}_{i}(\boldsymbol{z}) \boldsymbol{\psi}_{i}\left(\boldsymbol{z}^{\prime}\right),
$$

which are in practice obtained by solving the homogeneous Fredholm integral equation of the second kind:

$$
\int_{\Omega} \boldsymbol{\Gamma}_{x}\left(\boldsymbol{z}, \boldsymbol{z}^{\prime}\right) \boldsymbol{\psi}_{i}\left(\boldsymbol{z}^{\prime}\right) \mathrm{d} \boldsymbol{z}^{\prime}=\lambda_{i} \boldsymbol{\psi}_{i}(\boldsymbol{z}) .
$$

Since $\boldsymbol{\Gamma}_{x}\left(\boldsymbol{z}, \boldsymbol{z}^{\prime}\right)$ is bounded, symmetric and positive semi-definite, and furthermore in most practical cases can be assumed positive definite, these eigenvalues $\lambda_{i}$ are non-negative and the eigenfunctions $\boldsymbol{\psi}_{i}(\boldsymbol{z})$ satisfy the following orthogonality condition:

$$
\left\langle\boldsymbol{\psi}_{i}(\boldsymbol{z}), \boldsymbol{\psi}_{j}(\boldsymbol{z})\right\rangle=\int_{\Omega} \boldsymbol{\psi}_{i}(\boldsymbol{z}) \boldsymbol{\psi}_{j}(\boldsymbol{z}) \mathrm{d} \boldsymbol{z}=\delta_{i j}
$$

with $\delta_{i j}$ the Kronecker delta and $\langle\cdot, \cdot\rangle: \Omega \times \Omega \mapsto \mathbb{R}$ an inner product space. Hence, the eigenfunctions form a complete orthogonal basis on an $\mathcal{L}_{2}$ Hilbert space. In this case, the series expansion in Eq. (3) can be shown to be optimally convergent [31].

To limit the computational cost, the series expansion in Eq. (1) is usually truncated by retaining only the $T \in \mathbb{N}^{+}$largest eigenvalues and corresponding eigenfunctions of $\boldsymbol{\Gamma}_{x}\left(\boldsymbol{z}, \boldsymbol{z}^{\prime}\right)[32$, which yields an optimal series expansion with respect to the global mean squared error [33. Formally, this is expressed as

$$
\tilde{x}(\boldsymbol{z}, \theta)=\mu_{x}(\boldsymbol{z})+\sigma_{x} \sum_{i=1}^{T} \sqrt{\lambda_{i}} \boldsymbol{\psi}_{i}(\boldsymbol{z}) \xi_{i}(\theta) .
$$

The variance of $\tilde{x}(\boldsymbol{z}, \theta)$ can be expressed as:

$$
\sigma_{x}^{2}\{\tilde{x}(\boldsymbol{z}, \theta)\}=\sigma_{x}^{2} \sum_{i=1}^{T} \lambda_{i} \boldsymbol{\psi}_{i}^{2}(\boldsymbol{z}) .
$$

As such, the error of the variance

$$
\epsilon(\boldsymbol{z})=1-\frac{\sigma_{x}^{2}\{\tilde{x}(\boldsymbol{z}, \theta)\}}{\sigma_{x}^{2}}
$$

is given by

$$
\epsilon(\boldsymbol{z})=1-\sum_{i=1}^{T} \lambda_{i} \boldsymbol{\psi}_{i}^{2}(\boldsymbol{z}),
$$

and the mean error of the variance is defined as

$$
\bar{\epsilon}(\boldsymbol{z})=1-\frac{1}{|\Omega|} \sum_{i=1}^{T} \lambda_{i} \int_{\Omega} \boldsymbol{\psi}_{i}^{2}(\boldsymbol{z}),
$$


with $|\Omega| \in \mathbb{R}_{\alpha}^{+}$the size of the physical domain. From this equation, it is clear that the random field representation following the truncated KL expansion inherently underestimates the actual variance of the original random field. Furthermore, since the variance error varies over the model domain, the truncated KL random field is only approximately homogeneous.

In this paper, the case of isotropic Gaussian random fields that are governed by an exponential autocorrelation kernel is considered:

$$
\boldsymbol{\Gamma}_{x}\left(\boldsymbol{z}, \boldsymbol{z}^{\prime}\right)=\exp \left\{-\frac{\left|\boldsymbol{z}-\boldsymbol{z}^{\prime}\right|}{L}\right\}
$$

with $L \in \mathbb{R}_{0}^{+}$the correlation length of the random field ${ }^{2}$. This quantity represents the decay of the autocorrelation over distance. In the limit case of $L \rightarrow \infty$ all points of the random field are perfectly correlated, and the field reduces to a random variable. Conversely, when $L \rightarrow 0$, an independent random variable is defined for each location in $\Omega$.

In the particular case of Eq. 11), an exact analytic solution to the integral eigenvalue problem, introduced in Eq. (4) exists 31. Following this approach for a domain $\Omega \subset \mathbb{R}^{1}$, the eigenvalues are given by:

$$
\lambda_{i}=\frac{2 c}{\omega_{i}^{2}+c^{2}}
$$

and

$$
\lambda_{i}^{*}=\frac{2 c}{\omega_{i}^{* 2}+c^{2}},
$$

where $\omega_{i}$ for $i$ odd and $\omega_{i}^{*}$ for $i$ even are solutions to the transcendental equations:

$$
c-\omega \tan (\omega|\Omega|)=0,
$$

and

$$
\omega^{*}+c \tan \left(\omega^{*}|\Omega|\right)=0
$$

with $|\Omega| \in \mathbb{R}_{\alpha}^{+}$the length of the 1-dimensional domain and $c=1 / L$. Similarly, the eigenfunctions can be determined as:

$$
\psi_{i}(z)=\frac{\cos \left(\omega_{i} z\right)}{\sqrt{|\Omega|+\frac{\sin \left(2 \omega_{i}|\Omega|\right)}{2 \omega_{i}}}},
$$

and

$$
\psi_{i}^{*}(z)=\frac{\sin \left(\omega_{i}^{*} z\right)}{\sqrt{|\Omega|-\frac{\sin \left(2 \omega_{i}^{*}|\Omega|\right)}{2 \omega_{i}^{*}}}} .
$$

For 2-dimensional rectangular problems with separable autocorrelation function the solution of Eq. (4) can be determined from the 1-dimensional solutions [17:

$$
\lambda_{i}=\lambda_{i, z_{1}}^{1 \mathrm{D}} \lambda_{i, z_{2}}^{1 \mathrm{D}},
$$

\footnotetext{
${ }^{2}$ These fields are also called Ornstein-Uhlenbeck processes.
} 
and

$$
\psi_{i}(\boldsymbol{z})=\psi_{i}^{1 \mathrm{D}}\left(z_{1}\right) \psi_{i}^{1 \mathrm{D}}\left(z_{2}\right) .
$$

Note that an analytic solution is only available for this special case. To gain an overview of numeric solutions applicable in general, the reader is advised to the literature, e.g. [17] or [24]. Furthermore, it should be noted that by introducing a regular exponential kernel, non-smooth sample paths are inherently included in the analysis as a result of the non-differentiability of Eq. (11) at $\boldsymbol{z}=\boldsymbol{z}^{\prime}$ 33. Differentiable alternatives, e.g. the linear exponential, the squared exponential or the Whittle-Matérn autocorrelation function, provide a faster convergence with less truncation terms $T$ [34, 35. For a recent study on the autocorrelation function and the corresponding sample path smoothness affecting the results of a random field analysis, the reader is referred to 35. In this study however, the availability of analytic solutions to Eq. (4) outweighs possible effects of the non-smoothness of the sample paths on the computations.

\subsection{Epistemic uncertainty: interval fields}

Similar to a finite-dimensional random field, a finite-dimensional interval field $\boldsymbol{x}^{I}(\boldsymbol{z})$ describes a set of dependent intervals $x^{I} \in \mathbb{R}^{n_{b}}\left(z_{i}\right)$. Here, $\mathbb{I} \mathbb{R}$ is the space of real-valued intervals, which are assigned to a countable number of locations $z \in \Omega$ in the spatial domain. Following the explicit formulation introduced in [36, an interval field $\boldsymbol{x}^{I}(\boldsymbol{z}): \Omega \times \mathbb{R}^{n_{b}} \mapsto \mathbb{R}$ is specifically defined as:

$$
\boldsymbol{x}^{I}(\boldsymbol{z})=\sum_{i=1}^{n_{b}} \boldsymbol{\psi}_{i}(\boldsymbol{z}) \alpha_{i}^{I}
$$

and can be interpreted as the superposition of $i=1, \ldots, n_{b} \in \mathbb{N}$ base functions $\boldsymbol{\psi}_{i}(\boldsymbol{z}): \Omega \mapsto[0,1]$. Those represent a set of spatial uncertainty patterns, scaled by independent interval scalars $\alpha_{i}^{I} \in \mathbb{I} \mathbb{R}$, which represent the magnitude of the uncertainty. Note that in general, these base functions are not orthogonal by construction:

$$
\left\langle\boldsymbol{\psi}_{i}(\boldsymbol{z}), \boldsymbol{\psi}_{j}(\boldsymbol{z})\right\rangle=\int_{\Omega_{e}} \boldsymbol{\psi}_{i}(\boldsymbol{z}) \boldsymbol{\psi}_{j}(\boldsymbol{z}) \neq \delta_{i j},
$$

which affects the modeling of the dependence between interval scalars 37. Indeed, the dependence between intervals $x^{I} \in \mathbb{R}^{n_{b}}\left(z_{i}\right)$ at locations $z_{i}$ is controlled by the non-orthogonality of the base functions. Furthermore, since the interval scalars $\alpha_{i}^{I}$ remain fully independent, typically applied propagation schemes for interval analysis still can be applied [38. For a more complete overview of interval- and interval field methods, the reader is kindly referred to a recent review paper [26].

\section{Hybrid approaches to model mixed epistemic and aleatory uncertainties}

Naturally, also combinations of aleatory and epistemic uncertainty can be present in the description of the uncertain property. One possibility to model mixed uncertain parameters is given by the probability box (p-box) approach [4, the main idea of which is introduced in the following section. 
Considering uncertainty on the governing parameters of a random field, the p-box concept needs to be extended. The resulting framework of imprecise random fields, introduced separately by the authors in 39. and [23], in this case proves to be a valuable tool. The approach is summarized in Section 3.2 and applied in Section 5

\subsection{Probability box approach}

A straightforward approach to capture mixed, aleatory and epistemic, uncertainties is given by a probability box (p-box). A p-box $\left[\bar{F}_{x}, \underline{F}_{x}\right]$ is minimally defined by a left and right bound, $\bar{F}_{x}(x)$ and $\underline{F}_{x}(x)$ respectively, which is also denoted a distribution-free p-box. The p-box can be further described by the quintuple $\left(\bar{F}_{x}, \underline{F}_{x}, \mu_{x}^{I}, \sigma_{x}^{I}, \mathcal{F}\right)$ which has further information on, e.g. the confidence interval of the mean value $\mu_{x}^{I} \in \mathbb{I}$ holding

$$
\int_{-\infty}^{\infty} x \mathrm{~d} F(x) \in \mu_{x}^{I},
$$

the confidence interval $\left(\sigma_{x}^{2}\right)^{I} \in \mathbb{I}$ of the variance such that

$$
\left(\int_{-\infty}^{\infty} x^{2} \mathrm{~d} F(x)\right)-\left(\int_{-\infty}^{\infty} x \mathrm{~d} F(x)\right)^{2} \in\left(\sigma_{x}^{2}\right)^{I}
$$

\subsection{Imprecise random fields}

In Section 2.1, random field analysis has been introduced as a rigorous method to model spatially uncertain model properties in an aleatory framework. However, in a general engineering context, describing the quantities that are required to represent a random field crisply is often non-trivial or even impossible,

\footnotetext{
${ }^{3}$ Although depicted in one line here for reasons of clarity, please note that $\hat{F}_{x}$ is not necessarily equal to $\hat{x}$.
} 

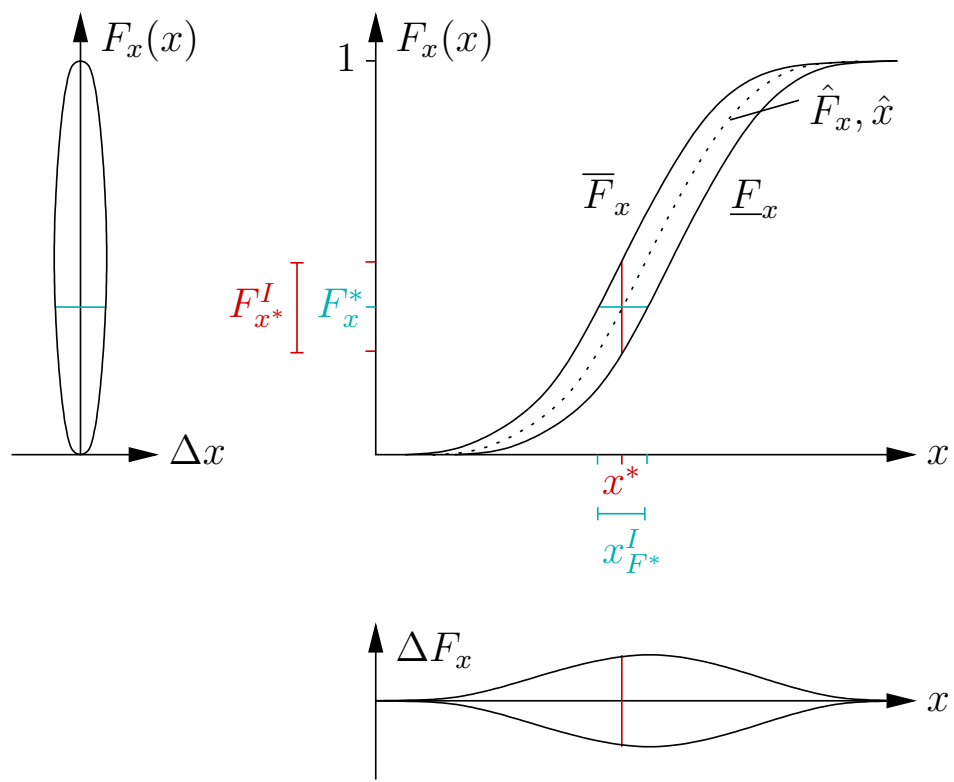

Figure 1: P-box visualized classically (top right) and as the deviation $\Delta x=x-\hat{x}$ from the midpoint line $\hat{x}$ of the interval $x_{F *}^{I}$ (left) as well as the deviation $\Delta F_{x}=F_{x}-\hat{F}_{x}$ from the midpoint line $\hat{F}_{x}$ of the interval $F_{x^{*}}^{I}$ (bottom).

given the omnipresent constraints on data availability. This observation led to the so-called concept of imprecise random fields (see e.g., [40, 39, 20, 23]), which form a generalization of p-boxes towards tempospatially uncertain quantities [4].

In the context of a random field $x(\boldsymbol{z}, \theta)$, given epistemic uncertainty on (some of) its hyper-parameters, the field becomes an imprecise random field $[x](\boldsymbol{z}, \theta)$. Since a random field requires to define a correlation random field is defined by the sextuplet $\left(\bar{F}, \underline{F}, \mu_{x}^{I}, \sigma_{x}^{I}, \mathcal{F}, \mathcal{C}\right)$, with $\mathcal{C}$ the set of admissible correlation functions. In case a predefined correlation function is selected that is parameterized by an appropriate correlation length $L$, the definition of an imprecise autocorrelation boils down to selecting appropriate bounds for $L$, and the sextuplet becomes $\left(\bar{F}, \underline{F}, \mu_{x}^{I}, \sigma_{x}^{I}, \mathcal{F}, L^{I}\right)$. The truncated KL expansion of an imprecise random field 205 in this case reads:

$$
[x](\boldsymbol{z}, \theta)=\mu_{x}^{I}(\boldsymbol{z})+\sigma_{x}^{I} \sum_{i=1}^{T} \sqrt{\lambda_{i}^{I}} \boldsymbol{\psi}_{i}^{I}(\boldsymbol{z}) \xi_{i}(\theta),
$$

with $\lambda_{i}^{I} \in \mathbb{R}^{+}$a set of strictly positive interval valued eigenvalues and $\boldsymbol{\psi}_{i}^{I}(\boldsymbol{z}): \Omega \times \mathbb{R} \mapsto \mathbb{R}$ interval fields representing the bounds on the corresponding eigenfunctions. It can therefore be understood that an imprecise random field describes a set of correlated p-boxes $[x](\theta)$ for each location $\boldsymbol{z} \in \Omega$. As such, when considering a single location $\boldsymbol{z}_{j} \in \Omega$, bounds for the cumulative distribution function (CDF) are locally given. Similarly, for a given $\theta_{j}$, also realizations $[x]\left(\boldsymbol{z}, \theta_{j}\right)$ are generated. The main difference with the 
realizations of a regular random field is that in case $\theta$ is fixed to a value $\theta_{j}$, the realizations become interval field valued:

$$
[x]\left(\boldsymbol{z}, \theta_{j}\right)=\mu_{x}^{I}(\boldsymbol{z})+\sigma_{x}^{I} \sum_{i=1}^{T} \sqrt{\lambda_{i}^{I}} \boldsymbol{\psi}_{i}^{I}(\boldsymbol{z}) \xi_{i}\left(\theta_{j}\right) .
$$

Indeed, since epistemic uncertainty is present in the definition of $\mu_{x}, \sigma_{x}$ and $L$, a full admissible set of realizations that are consistent with these intervals is provided. It should be noted that, in case $\mathcal{F}$ contains also non-Gaussian distributions, the imprecise random field inherently also extends towards non-Gaussian random fields. Hence, the same considerations concerning the correlation and dependence of $\xi_{i}$ have to be made as for regular random fields. Specifically, Eq. (2) has to be solved in this case explicitly for each class of non-Gaussian distribution functions in $\mathcal{F}$. This, however, falls outside the scope of this paper.

Concerning the propagation of an imprecise random field $[x](\boldsymbol{z}, \theta)$ with an autocorrelation function $\boldsymbol{\Gamma}_{x}\left(\boldsymbol{z}, \boldsymbol{z}^{\prime} \mid L\right)$ through a monotonic model, the approach presented in [23] is applied. Let $\mathcal{G}(\Omega, L): \Omega \times L \mapsto$ $\{\boldsymbol{\lambda}, \boldsymbol{\psi}(\boldsymbol{z})\}$ denote the process of solving Eq. (4) for $m$ eigenpairs of $\boldsymbol{\Gamma}_{x}$ given a crisp value. Then, those values for $L$ that yield extreme values in $\sqrt{\lambda_{i}} \psi_{i}(\boldsymbol{z})$ are determined following an optimization approach:

$$
\begin{aligned}
& \underline{L}_{i}^{*}=\underset{\mathcal{G}(L)}{\arg \min }\left\|\sqrt{\lambda_{i}} \psi_{i}(\boldsymbol{z})\right\|_{2}, \quad \text { s.t. } L \in L^{I} \\
& \bar{L}_{i}^{*}=\underset{\mathcal{G}(L)}{\arg \max }\left\|\sqrt{\lambda_{i}} \psi_{i}(\boldsymbol{z})\right\|_{2}, \quad \text { s.t. } L \in L^{I}
\end{aligned}
$$

with $i=1, \ldots, T$. The underlying idea is to look for those $L$ that correspond to extrema in the $\mathcal{L}_{2}$ norm of the basis function in each random field mode. As such, a complete bounding set for the imprecise random field basis is obtained, while preserving all realizations of the base functions to be orthogonal. Furthermore, due to the $\mathcal{L}_{2}$ norm being differentiable, the objective function yields a smooth, convex, non-linear optimization problem in limited dimension. The at most $2 T$ solutions are then concatenated in a single vertex set $\mathcal{A}$ :

$$
\mathcal{A}=\left\{\underline{L}_{1}^{*}, \bar{L}_{1}^{*}, \underline{L}_{2}^{*}, \bar{L}_{2}^{*}, \ldots \underline{L}_{T}^{*}, \bar{L}_{T}^{*}\right\},
$$

and the eigenpairs $\{\boldsymbol{\lambda}, \boldsymbol{\psi}(\boldsymbol{z})\}$ are computed using Eq. (4) for each of these $L \in \mathcal{A}$. The objective functions of this optimization are visualized in Figure 2, where the eigenvalues $\lambda_{1}, \ldots, \lambda_{7}$ are depicted as a function of the correlation length $L$, both normalized by the domain length $l$. Except for $\lambda_{1} / l$ which converges to one, the eigenvalues are not monotonically increasing but decay towards zero after reaching a maximum turning point. As can be understood from Eq. (26), this procedure corresponds to a maximization of the variance of the random field at the input side by actively looking for those combinations that bound the KL basis. The values $L_{i}^{*} \in L^{I}$ are called intermediate values in the following.

Concerning the actual propagation of the imprecise random field, let $\mathcal{M}$ be a deterministic numerical model that is used to approximate $\boldsymbol{y} \in \mathbb{R}^{d_{s}}$, which is the solution of a (set of) differential equations. The operator works through a set of (usually) real-valued function operators $\boldsymbol{g}=\left\{g_{i} \mid i=1, \ldots, d_{s}\right\}$ :

$$
\mathcal{M}(\boldsymbol{x}): \boldsymbol{y}=g_{i}(\boldsymbol{x}), \quad g_{i}: \mathbb{R}^{d_{i}} \mapsto \mathbb{R}, i=1, \ldots, d_{s},
$$




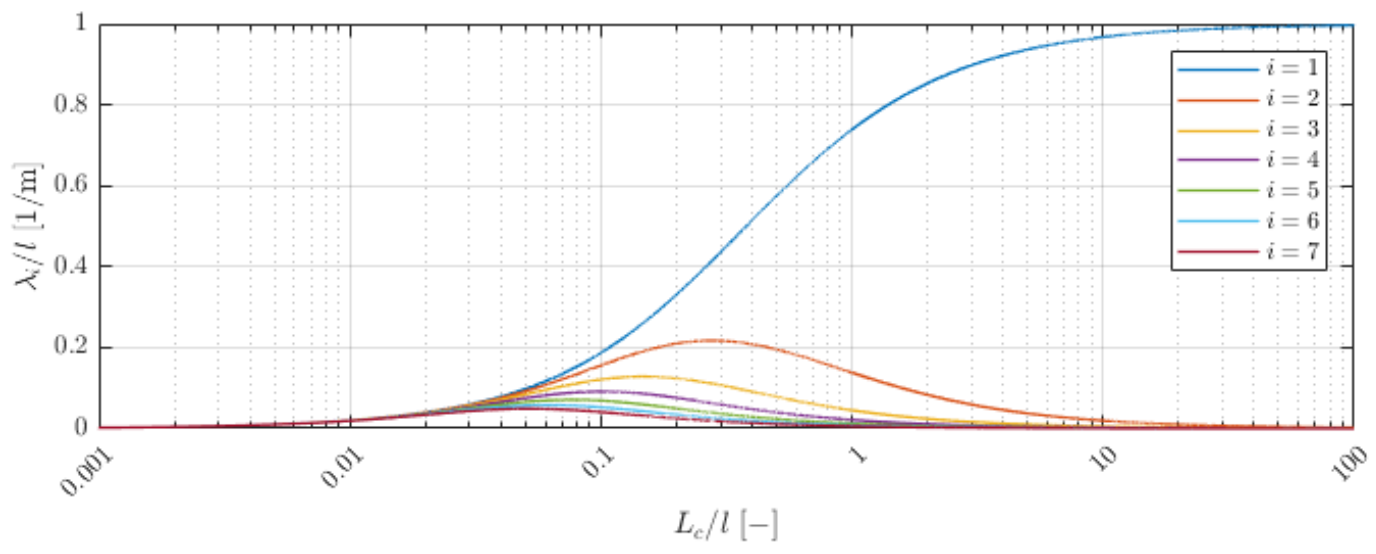

(a) Convergence of $\lambda_{1} / l$ towards one while $\lambda_{i} / l, i>1$ decays towards zero after reaching a maximum turning point.

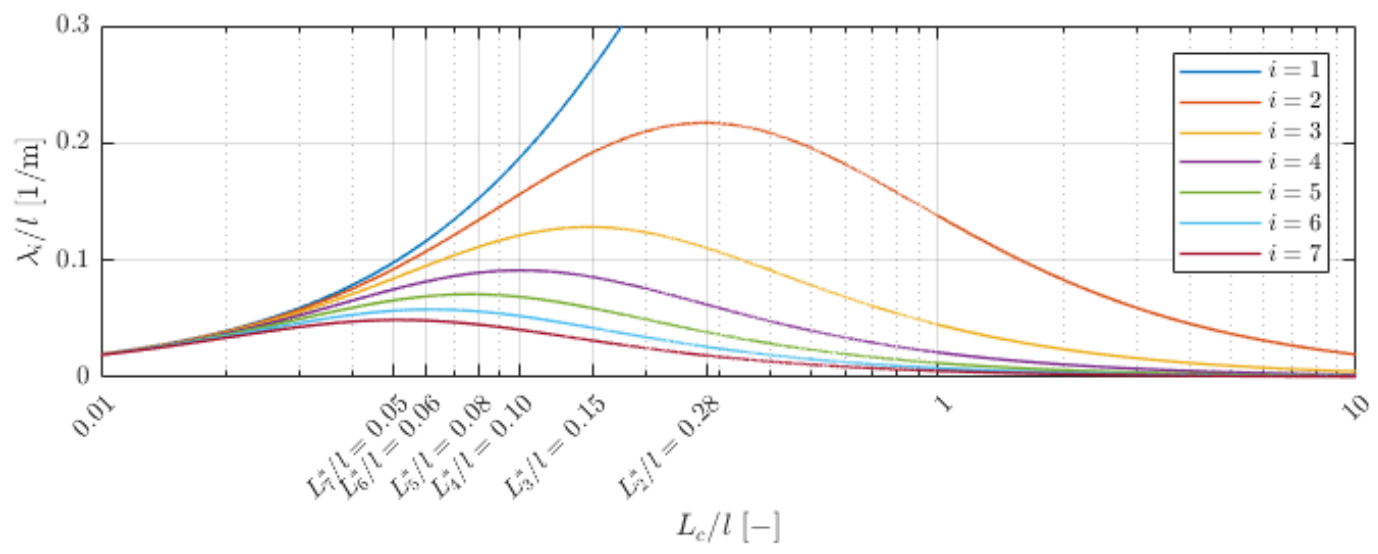

(b) Intermediate correlation lengths $L_{i}^{*}, i=2, \ldots, 7$ corresponding to the maximum turning point eigenvalue $\lambda_{i}$.

Figure 2: Eigenvalues $\lambda_{i}, i=1, \ldots, 7$ as a function of the correlation length $L$, both normalized by domain length $l$.

with $\boldsymbol{x} \in \mathcal{F} \subset \mathbb{R}^{d_{i}}$ the vector of model parameters and $\mathcal{F}$ the sub-domain of feasible parameters (e.g., nonnegative contact stiffness). Since each realization of a correlation length in $\mathcal{A}$ corresponds to a crisp random field, they can readily be propagated towards a probabilistic quantity of interest at the response side of the model using typically available simulation methods such as Monte Carlo simulation, Subset Sampling [41, Line Sampling [42] or in case of dynamical time domain problems, Directional Importance Sampling [43].

In this case, we are interested in determining the p-box on $[\boldsymbol{y}]=\left[\bar{F}_{y}(\boldsymbol{y}), \underline{F}_{y}(\boldsymbol{y})\right]$. In case the responses $\boldsymbol{y}$ behave strictly monotonically with respect to the sample path realizations of $[x]\left(\boldsymbol{z}, \theta_{j}\right)$, the typically applied double-loop procedure, where the reliability problem has to be solved for each step of a global optimization algorithm that actively looks for those $\left\{L_{i}\right\}$ that bound $[\boldsymbol{y}]$, can be replaced by a more simple propagation scheme. Namely, in this case, the left bound of the p-box of the model response $y_{k}$ is defined as

$$
\underline{F}_{y}\left(y_{k}\right)=\min _{\left\{L_{i}\right\} \in\{\mathcal{A}\}} F_{y_{k}}\left(\mathcal{M}\left(x\left(\boldsymbol{z}, \theta_{j}, L_{i}\right)\right)\right), \quad k=1, \ldots, n_{y}
$$


where the set $\mathcal{A}$ is determined a priori using Eq. 26). $F_{y_{k}}$ denotes the CDF corresponding to the response that results from propagating one realization of the imprecise random field $[x](\boldsymbol{z}, \theta, L)$ - i.e., a random field $x\left(\boldsymbol{z}, \theta_{j}, L_{i}\right)$ - through the model $\mathcal{M}$ and $n_{k}$ is the number of responses of interest. The corresponding right bound is defined as

$$
\bar{F}_{y}\left(y_{k}\right)=\max _{\left\{L_{i}\right\} \in\{\mathcal{A}\}} F_{y_{k}}\left(\mathcal{M}\left(x\left(\boldsymbol{z}, \theta_{j}, L_{i}\right)\right)\right), \quad k=1, \ldots, n_{y} .
$$

Note that monotonicity with respect to sample path realizations is a rather stringent condition. For instance, when considering an imprecise stochastic process load in a structural dynamics problem, the case could occur where maximizing the variance at the input side does not lead to an extreme response, since in this case, also the dominant frequency of the load should match the natural frequencies of the structural model (see e.g., 21] for an illustration of such a case). In more general cases, other propagation schemes for imprecise probabilities, such as NISS [12, 13] or the more recently introduced Operator Norm framework [21] should be applied. These methods, however, require further development aimed at estimating the full response p-box rather than a single quantity of interest such as the probability of failure.

\section{Application to non-linear material behaviour: brittle damage of a concrete beam}

The propagation of imprecise random fields using the optimization approach given by Eq. 26 has been investigated in terms of linear problems in [23. Solving non-linear differential equations with uncertain input parameters, however, implies further challenges. One important non-linearity in FE analyses in structural mechanics is material non-linearity, such as plasticity and damage. In concrete structures, consisting mostly of quasi-brittle material, continuum damage mechanics is often used to describe the decrease in load bearing capacity of the structure leading to failure. Uncertainties in the continuum damage mechanics models are also an important aspect as the models often rely on material properties which are not easily obtained. As mean value and standard deviation of material parameters usually can be obtained by experiments, these model parameters are thought of as an aleatory uncertainty within this paper. On the other hand, the correlation length of the correlation function can hardly be determined. For this reason, it is assumed to be an epistemic uncertainty and modelled by an interval.

In this section, the mechanical model underlying the studies within this paper is introduced. Furthermore, the difficulties occurring when propagating imprecise random fields within an FE analysis are discussed. This concerns especially the truncation error resulting from different correlation lengths. Based on this section, the importance of intermediate correlation lengths is finally investigated in Section 5

\subsection{Model description}

In order to investigate the impact of uncertainties in a structural mechanics problem, a four-point bending test of a concrete beam is analyzed using a non-intrusive stochastic FE approach. The structure geometry is 
depicted in Figure 3, where the dimensions are taken as $l=10 \mathrm{~m}$ and $h=1 \mathrm{~m}$. The model is discretized by $80 \times 8$ linear four-node elements and loaded by two forces of each $F=4000 \mathrm{kN}$ at $z_{1}=3.5 \mathrm{~m}$ and $z_{1}=6.5 \mathrm{~m}$, respectively. The model is two-dimensional and a plane strain assumption is used. The elastic properties of the model are supposed to be deterministic, with the Young's modulus $E=30 \frac{\mathrm{kN}}{\mathrm{mm}^{2}}$ and the Poisson's ratio $\nu=0.2$. The uncertain material parameters are assumed to be wholly on the continuum damage model parameters.

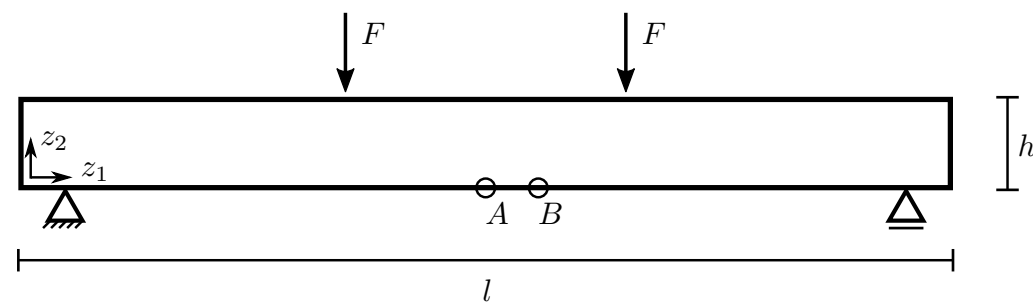

Figure 3: FE four point bending model, defining the point $A\left(z_{1}=5 \mathrm{~m}, z_{2}=0 \mathrm{~m}\right)$ and $B\left(z_{1}=5.5 \mathrm{~m}, z_{2}=0 \mathrm{~m}\right)$ which are the focus points of this study.

The loss of load bearing capacity of the model is given by a scalar damage parameter $D$ which is here taken as an isotropic, hence scalar, quantity. As the beam is subjected to increasing loads, micro-cracks and voids start to develop in the material. This physical phenomenon is here represented by the scalar damage parameter. Therefore, as this parameter increases with the increasing loads, the stiffness of the beam is decreased. This material model can be represented by the following Helmholtz free energy function $\psi$,

$$
\rho \psi=\frac{1}{2}(1-c D) \varepsilon: \mathbb{C}: \varepsilon
$$

where $\rho$ is the density, $c$ represents a closure parameter, $\varepsilon$ is the second order strain tensor and $\mathbb{C}$ the fourth order elasticity tensor. By this, with $\boldsymbol{\sigma}$ the second order stress tensor, the constitutive equation, of the model can be written as

$$
\boldsymbol{\sigma}=(1-c D) \mathbb{C}: \boldsymbol{\varepsilon}
$$

The closure parameter

$$
c= \begin{cases}h_{\mathrm{c}} & \text { if } \quad \operatorname{tr} \varepsilon<0, \\ 1 \quad \text { if } \quad \operatorname{tr} \varepsilon \geq 0 .\end{cases}
$$

is introduced in order to distinguish the behaviour of concrete in tension and compression, since under compression a part of the cracks close again and therefore a part of the stiffness is recovered. In Eq. (32), it is clear how an increased damage reduces the material response by reducing the elastic properties of the material.

Many different elastic damage laws have been developed over the last three decades for diverse applications and materials [4, 45, 46]. Here, a quasi-brittle damage model based on threshold $Y_{D}$ of the energy 
release rate $Y$ is implemented [47,44. The simplicity of this model allows for a comprehensive interpretation of the material parameters involved and fits well with the goal of this work to analyze imprecise random fields for a non-linear mechanical problem. The damage model for monotonically increasing loads used here is given by

$$
D=\left(\frac{Y_{\max }-Y_{D}}{r}\right)^{s}, \quad \text { when } Y_{\max }>Y_{D}
$$

where $1 / r$ controls the intensity of the damage rate. The material parameters $r, s$ and $Y_{D}$ are determined experimentally. In Eq. [34, $Y_{\max }$ is the maximum of the energy release rate $Y$ throughout the load history,

$$
Y=\frac{c}{2} \varepsilon: \mathbb{C}: \varepsilon
$$

The material parameters $s$ and $h_{\mathrm{c}}$ are assumed as deterministic and taken as $s=1$ and $h_{\mathrm{c}}=0.057$, respectively, as provided in [44]. The other two parameters, $Y_{D}$ and $r$, affect the solution space by defining starting point and intensity of the damage. Therefore, they are assumed as uncertain and described as imprecise random fields. For each interval bound and intermediate correlation length value, the random fields are discretizend by KL expansion, using the analytic solution given in Eq. 18] and Eq. (19).

\subsection{Sensitivity to damage parameters}

To asses the outcome of the damage distribution on the beam in the presence of both random fields, $Y_{D}$ and $r$, a sensitivity analysis of all uncertain material parameters is performed to accurately investigate the output distributions. The sensitivity analysis of the uncertain material parameters is carried out on the evolution of the damage function in Eq. (34) as a function of a load factor to the force $F$ given in Section 4.1 . In Figure 4, the damage evolution for each random variable independently and for both random variables combined are displayed. The influence of the threshold $Y_{D}$ for damage to occur on the damage evolution curve is limited to the load at which damage begins to evolve. On the other hand, the intensity $1 / r$ of the damage rate $\dot{Y}$ affects the solution space much more.

In the sensitivity analysis, the damage evolution of 1000 random samples were calculated for both random quantities, $r$ and $Y_{D}$. Furthermore, the combination of both quantities is sampled with 2000 random variables. The statistics of the final damage value, i.e. the quantity of interest, were computed with Monte Carlo simulation and are also displayed in Figure 4. From the results presented, it is evident that the randomness of the parameter $r$ has a larger influence on the final damage level than $Y_{D}$. However, although the distribution of the quantity of interest for two random variables simultaneously is mainly affected by $r$, adding $Y_{D}$ as second random input slightly increases the standard deviation of the distribution, see Table 1 The results presented here should be kept in mind when analyzing the CDFs of Section 5.1 . 


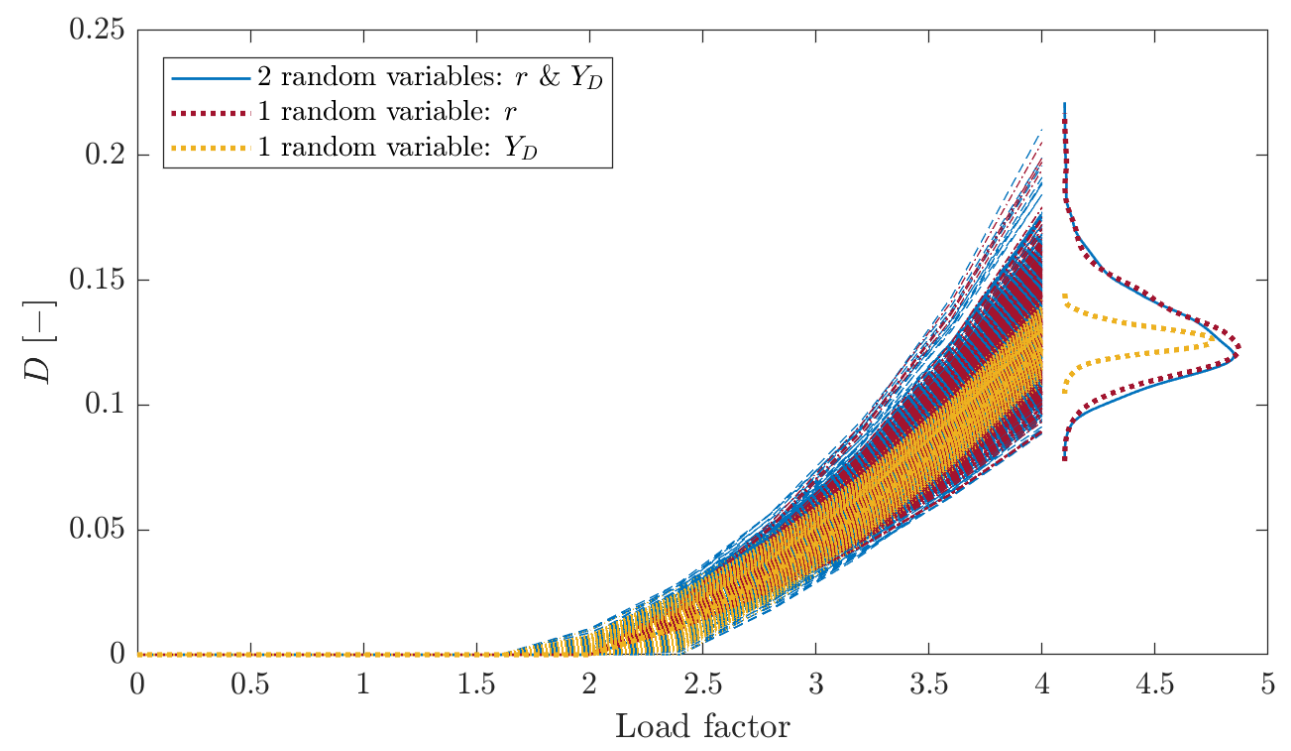

Figure 4: Sensitivity of the damage $D$ to the input random variables $r$ and $Y_{D}$, as well as the interference of both, sampled using 1000 Monte Carlo samples for one random variable input and 2000 Monte Carlo samples for two random variable inputs.

Table 1: Influence of $r$ and $Y_{D}$ on the distribution of the damage evolution.

\begin{tabular}{c|c|c|} 
& Mean value & Standard deviation \\
\hline One random variable $r$ & $\mu_{D}=0.1286$ & $\sigma_{D}=0.0158$ \\
One random variable $Y_{D}$ & $\mu_{D}=0.1261$ & $\sigma_{D}=0.0051$ \\
Two random variables $r$ and $Y_{D}$ & $\mu_{D}=0.1277$ & $\sigma_{D}=0.0166$ \\
\hline
\end{tabular}

\subsection{Truncation error of the KLE}

In order to propagate random fields based on different correlation lengths it is necessary to understand the impact of the KL series expansion truncation $T$ in Eq. (6) on the corresponding variance. For this purpose, the parameters of the imprecise random fields used in Section 5 are already reported in Table 2 . In a first simulation S1 two imprecise random fields $\left[Y_{D}\right]$ and $[r]$ are considered, both implying an interval valued correlation length $L_{\bullet}^{I} \in[2,16] \mathrm{m}$ to model a large epistemic uncertainty. In this case, the intermediate value $L_{\bullet, 2}^{*}=2.80 \mathrm{~m}$ is acquired by Eq. 26 under this interval constraint. The second simulation S2 considers the imprecise random field $[r]$ to be the only uncertain parameter. Here, the interval $L_{r}^{I} \in[1,2] \mathrm{m}$ considers a small epistemic uncertainty and is chosen such that the intermediate value $L_{r, 3}^{*}=1.48 \mathrm{~m}$ is located effectively near the midpoint of the interval. Note that the interval $L_{r}^{I} \in[1,2] \mathrm{m}$ actually contains a second intermediate value $L_{\bullet, 4}^{*}=1.0075 \mathrm{~m}$. It is, however, very close to the lower interval bound $\underline{L}_{r}=1 \mathrm{~m}$ and is neglected for the purpose of investigating the influence of an intermediate value located in the middle of an interval. 
Table 2: Material parameters described by imprecise random fields

\begin{tabular}{|c|c|c|c|c|}
\hline & \multirow[t]{2}{*}{ Mean value } & \multirow[t]{2}{*}{ Standard deviation } & \multicolumn{2}{|c|}{ Correlation length } \\
\hline & & & Interval & Intermediate value \\
\hline $\begin{array}{ll} & {\left[Y_{D}\right]} \\
\mathrm{S} 1 & {[r]} \\
\end{array}$ & $\begin{aligned} \mu_{Y_{D}} & =0.02 \mathrm{MPa} \\
\mu_{r} & =0.5 \mathrm{MPa}\end{aligned}$ & $\begin{aligned} \sigma_{Y_{D}} & =0.004 \mathrm{MPa} \\
\sigma_{r} & =0.1 \mathrm{MPa}\end{aligned}$ & $L_{\bullet}^{I} \in[2,16] \mathrm{m}$ & $L_{\bullet}^{*}=2.80 \mathrm{~m}$ \\
\hline $\mathrm{S} 2 \quad[r]$ & $\mu_{r}=0.5 \mathrm{MPa}$ & $\sigma_{r}=0.1 \mathrm{MPa}$ & $L_{r}^{I} \in[1,2] \mathrm{m}$ & $L_{r}^{*}=1.48 \mathrm{~m}$ \\
\hline
\end{tabular}

In Figure 5 the mean variance error $\bar{\epsilon}(\boldsymbol{z})$, determined by Eq. $(10)$, of a 2-dimensional random field is depicted as a function of the truncation order $T$ for the correlation length ratios $L / l$ of interest.

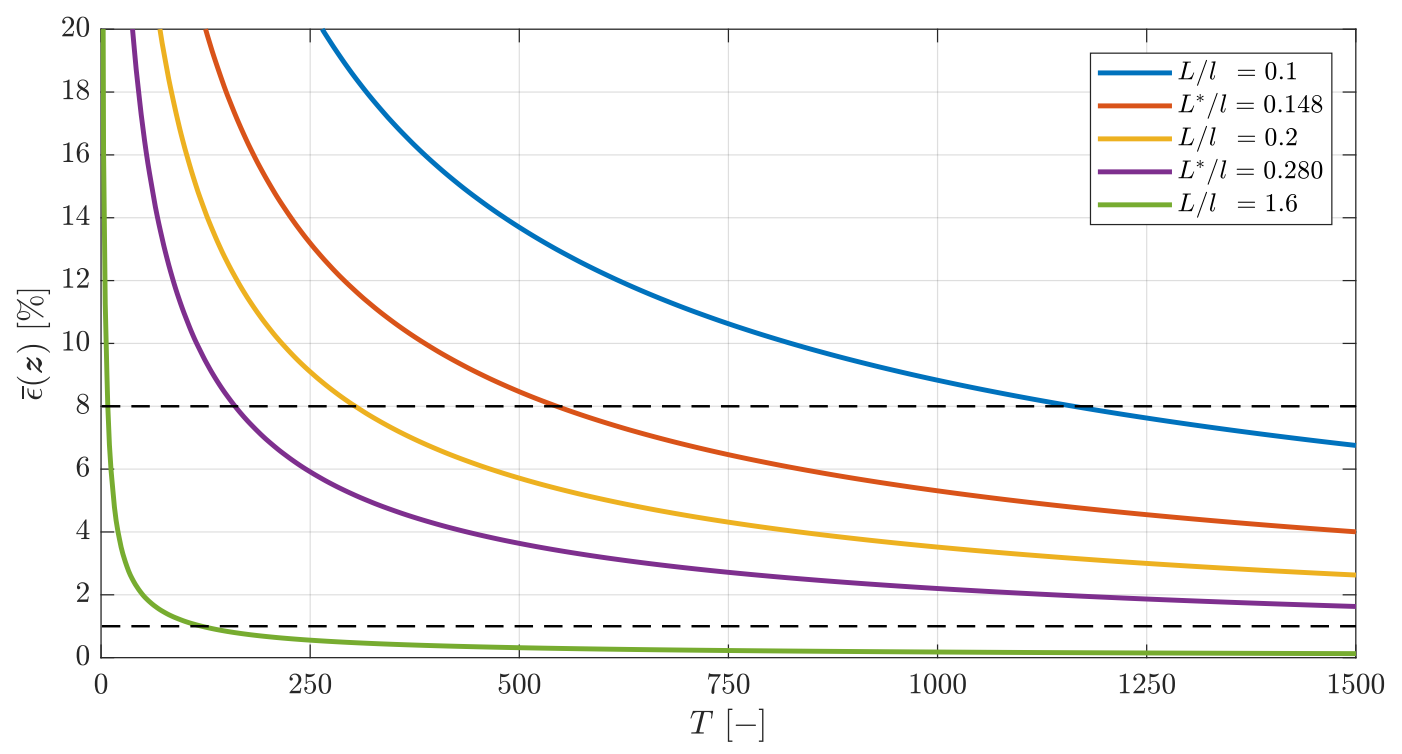

Figure 5: Mean variance error $\bar{\epsilon}(\boldsymbol{z})$ of the chosen correlation lengths depending on the truncation order $T$. for ratios $L / l \ll 1$. To ensure that the p-box is not attributed to a differing precision of the variance, the truncation is chosen corresponding to a constant mean variance error. For this, the following considerations are taken into account:

1. To keep S1 and S2 comparable, the mean variance error is chosen to be the same for both simulations.

2. The truncation order $T$ has to be chosen as to reduce the stochastic dimension, while maintaining a reasonably low fluctuation of the local variance.

Note that considering two random fields, the stochastic dimension is twice the truncation order. For this reason, the mean variance error is chosen to be $\bar{\epsilon}(\boldsymbol{z})=8 \%$ with regard to the computational expense. This 
value can be changed arbitrarily based on the available computational resources. The truncation orders corresponding to a 2-dimensional random field with the chosen error are listed in Table 3 for the chosen ratios $L / l$.

Table 3: Truncation orders corresponding to the chosen variance error $\bar{\epsilon}(\boldsymbol{z})=8 \%$

\begin{tabular}{c|c|c|c|c|c|}
\hline Correlation length & $L / l=0.1$ & $L^{*} / l=0.148$ & $L / l=0.2$ & $L^{*} / l=0.280$ & $L / l=1.6$ \\
\hline Truncation order & $T=1162$ & $T=544$ & $T=301$ & $T=159$ & $T=8$ \\
\hline
\end{tabular}

In Figure 6a the variance $\sigma_{x}^{2}$ distributions along the beam length $l$ are depicted corresponding to the chosen truncation orders. It can be seen that fulfilling the second demand on the chosen truncation error becomes impossible for large correlation length intervals: although the mean variance error is the same for each $L / l$, the local variation of the variance corresponding to $L / l=1.6$ is very high compared to smaller ratios. This is a challenge that is inherently related to applications with large correlation length intervals, i.e. cases with different orders of magnitudes between the interval bounds. The effect is mirrored within the distribution of the locations where the maximum damage $D_{\max }$ occurs within a simulation using $n_{\mathrm{MC}}=20000$ realizations, as visualized in Figure 6b. The color plot depicts the mean damage distribution $D$ determined statistically from all realizations. It can be seen for both the largest and the smallest chosen ratios $L / l$ of $\mathrm{S} 1$ that damage occurs in the region of maximum tension, meaning in the region between the forces at the bottom of the beam. Considering a sufficiently large sample size, it can be assumed by physical reasoning that the maximum damage $D_{\max }$ occurs equally distributed within this pure bending region as the bending moment there is constant. The red circles visualize by their size how frequently $D_{\max }$ is located at a certain point. Regarding the small ratio $L / l=0.2, D_{\max }$ is mostly located - as expected - at the bottom of the beam and equivalently distributed within the region of the constant maximal bending moment. For the large ratio $L / l=1.6$, however, the maximum damage is clustering much more in the regions of the maximum local variance. 


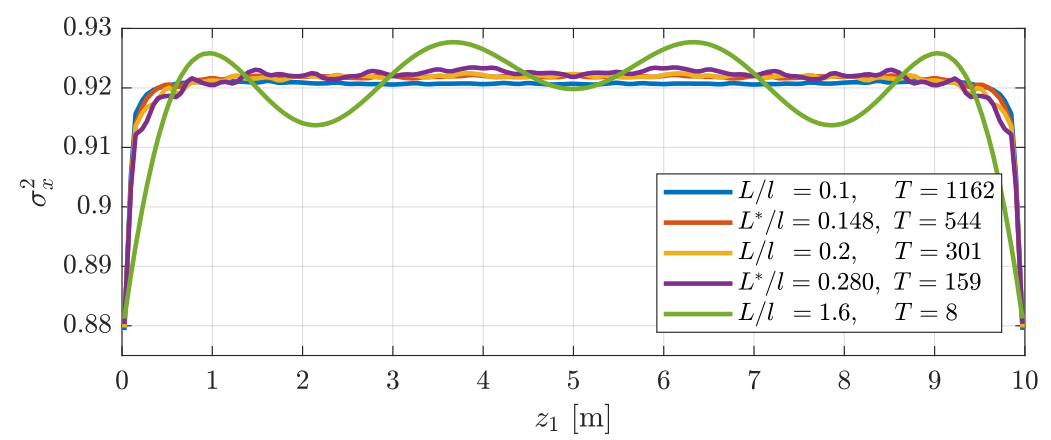

(a) Variance of the chosen correlation lengths corresponding to its truncation order leading to a mean variance error $\bar{\epsilon}(\boldsymbol{z})=8 \%$.

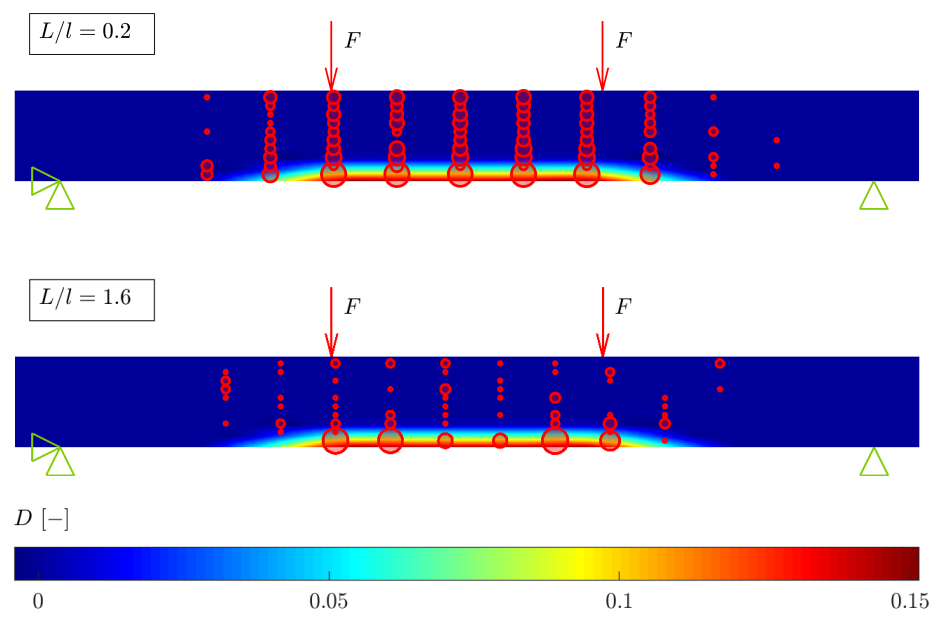

(b) Distribution of maximum damage $D_{\max }$ occurrence over the mean damage field, using $n_{\mathrm{MC}}=20000$ samples.

Figure 6: Influence of the local variance error $\epsilon(\boldsymbol{z})$ on the occurrence of the maximum damage.

For large correlation length intervals, gaining both (i) the same mean variance and (ii) a comparable local variance for each correlation length is only possible when the truncation order is increased such that both errors decay to zero. This however leads to stochastic dimensions that have to be balanced against the computational cost. Therefore, while studying the influence of an interval valued correlation length, artifact effects due to a local or global variance error should be carefully considered and thoroughly analyzed. In this paper, the mean variance error is chosen to be equal for all correlation lengths, as the quantities of interest are more affected by the global variance field than by local artifacts. 


\section{Effect of imprecise random fields on the structural damage p-box}

Performing a stochastic FE analysis considering several imprecise random fields becomes computationally challenging, as all combinations of correlation lengths must be considered. Determining the crucial intermediate correlation lengths a-priori already reduces the computational cost by avoiding the need of discretizing the intervals, as it has been done in a similar study before [22. In the following studies, the influence of imprecise random fields on the propagation through a non-linear material framework is investigated using the optimized intermediate correlation lengths, acquired from Eq. 26). The results are compared to a pure vertex analysis in terms of conservativeness and computational cost.

Considering the artificial clustering of the maximum damage for large correlation lengths as discussed in Section 4.3, $D_{\max }$ is not an appropriate quantity of interest here. Instead, the global damage $D_{A}$ is investigated, which is obtained for each realization by integrating the damage over the whole domain $\Omega$ :

$$
D_{A}=\frac{\int_{\Omega} D \mathrm{~d} \Omega}{\int_{\Omega} \mathrm{d} \Omega}
$$

This way, the artifacts due to a locally varying variance error are smoothed out and $D_{A}$ can be connected to the mean variance error $\bar{\epsilon}(\boldsymbol{z})$, which is the same for all chosen correlation lengths by choosing a different truncation order.

As a second quantity of interest, the local damage $D$ is investigated at two points $A$ and $B$, both located at the bottom $\left(z_{2}=0 \mathrm{~m}\right)$ of the beam, see Figure 3 . While point $A$ is chosen at the center $\left(z_{1}=5 \mathrm{~m}\right)$ of the beam, point $B$ is chosen at $z_{1}=5.5 \mathrm{~m}$ where the local variances of all correlation length ratios are as similar as possible, see Figure 6a. However, note that $D$ depends on the whole strain field (compare Eq. (34) and Eq. (35p) and therefore incorporates the whole variance field. The quantities of interest are each determined and depicted as a p-box complemented by the deviations of the midpoint line, as proposed with Figure 1 .

In the following sections, two simulations are investigated. In Section 5.1 , the influence of the intermediate correlations lengths is investigated from an engineering point of view. As the epistemic uncertainty of a correlation length is usually high, a large interval $L_{\bullet}^{I} \in[2,16] \mathrm{m}$ is chosen for both imprecise random fields $\left[Y_{D}\right](\boldsymbol{z}, \theta)$ and $[r](\boldsymbol{z}, \theta)$. To ensure that the observed phenomena are not due to other reasons such as a lack of convergence or an interference of the two imprecise random fields, in Section 5.2 the study is adjusted such that the only uncertain parameter is the imprecise random field $[r](\boldsymbol{z}, \theta)$ whose correlation length interval $L_{r}^{I} \in[1,2] \mathrm{m}$ is relatively narrow. Here, the intermediate value $L_{r}^{*}=1.48 \mathrm{~m}$ lies central within $L_{r}^{I}$. Furthermore, the standard normal distributed random variables $\xi_{i}(\theta), i=1, \ldots, T$, to be inserted in Eq. (24), are sampled using the same seed.

\subsection{Simulation 1: Two imprecise random fields containing a large epistemic uncertainty}

Within this study, two imprecise random fields $\left[Y_{D}\right](\boldsymbol{z}, \theta)$ and $[r](\boldsymbol{z}, \theta)$ are considered affecting the nonlinear damage evolution. Both random fields are imprecise due to an interval valued correlation length 
$L_{\bullet}^{I}=[2,16] \mathrm{m}$ caused by a large epistemic uncertainty. The simulation is performed by Monte Carlo method using $n_{\mathrm{MC}}=40000$ samples in case both imprecise random fields are considered and $n_{\mathrm{MC}}=20000$ if there is only one imprecise random field input.

\subsubsection{Sensitivity of the global damage to the imprecise random field input parameters}

In Figure 7, the CDFs of the global damage for all correlation length combinations are compared to the simulations of using one imprecise random field only. For convenience, the results of the interval vertices are depicted in different colors while the CDFs containing intermediate correlation lengths are given in a grey dashed line. The mean $\mu_{D_{A}}$ and the standard deviation $\sigma_{D_{A}}$ corresponding to the CDFs resulting from each correlation length (combination) are further given in Table 4 . In there, the results of all correlation length combinations considering both imprecise random fields $[r]$ and $\left[Y_{D}\right]$ are given in the top left $3 \times 3$ entries. The right column contains the results assuming $r=\mu_{r}$ to be deterministic with $\left[Y_{D}\right]$ being an imprecise random field, while it is the other way round in the bottom row (both emphasized by a double bar separation). The global damage $D_{A}$ of a deterministic simulation with $r=\mu_{r}$ and $Y_{D}=\mu_{Y_{D}}$ is given in the right bottom cell for comparison. To quantify the effect of the correlation length to the mean $\mu_{D_{A}}$ and standard deviation $\sigma_{D_{A}}$, the maximum deviation of each quantity with respect to the mean quantity is obtained by

$$
\delta(\bullet)=\frac{\max (\bullet)-\min (\bullet)}{\operatorname{mean}(\bullet)}
$$

Note that the mean $(\bullet)$ is computed specifically for each simulation.

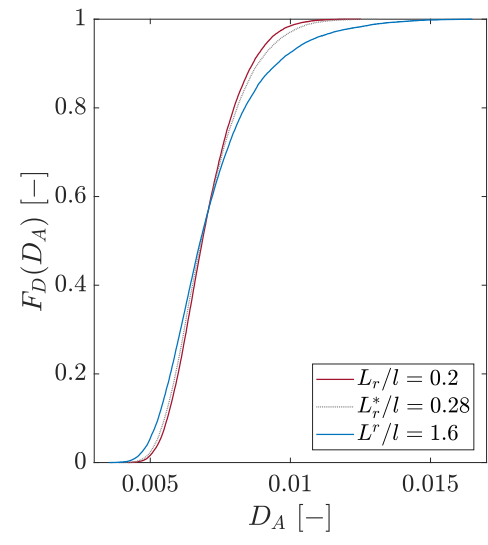

(a) Input: only $[r]$

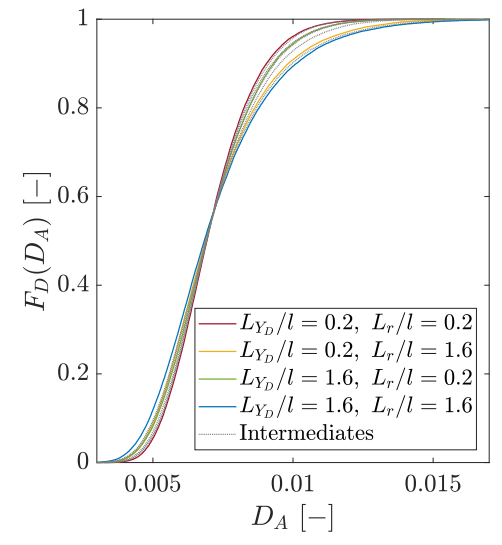

(b) Input: $[r]$ and $\left[Y_{D}\right]$

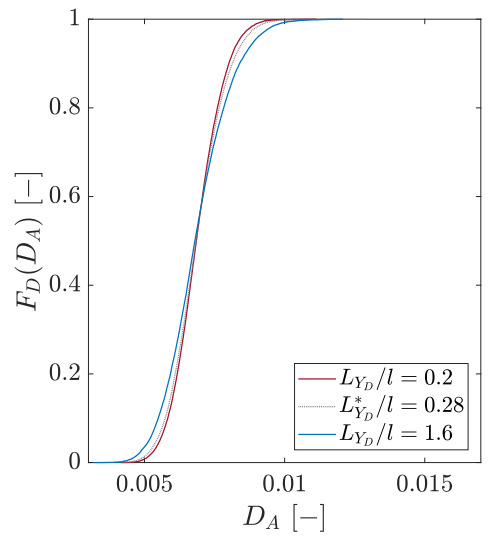

(c) Input: only $\left[Y_{D}\right]$

Figure 7: CDFs of the global damage $D_{A}$, resulting from simulations including one or two imprecise random field inputs.

It can be seen that the the correlation length has hardly an impact on the mean value $-\delta_{[r]}\left(\mu_{D_{A}}\right)=2.8 \%$, $\delta_{\left[Y_{D}\right]}\left(\mu_{D_{A}}\right)=0.0 \%$ and $\delta_{[r],\left[Y_{D}\right]}\left(\mu_{D_{A}}\right)=2.8 \%$ - although the mean $\mu_{D_{A}}$ slightly exceeds the global damage $D_{A}^{\text {det }}=0.0068$ resulting from a deterministic simulation. On the other hand, the standard deviation of $D_{A}$ 
is highly dependent on the correlation length. Here, the maximum deviation of the standard deviation with respect to the mean of all standard deviations is $\delta_{[r]}\left(\sigma_{D_{A}}\right)=41.9 \%$ and $\delta_{\left[Y_{D}\right]}\left(\sigma_{D_{A}}\right)=20.7 \%$ in case only

\section{Section 4.2 .}

Table 4: Mean value $\mu_{D_{A}}$ and standard deviation $\sigma_{D_{A}}$ of the global damage $D_{A}$ of the simulations including each correlation length combination of two imprecise random fields $[r]$ and $\left[Y_{D}\right]$ (top left $3 \times 3$ entries) as well as only one imprecise random field $[r]$, where $Y_{D}=\mu_{Y_{D}}$ is deterministic, (bottom row) or $\left[Y_{D}\right]$, where $r=\mu_{r}$ is deterministic, (right column) and the purely deterministic result assuming $r=\mu_{r}$ and $Y_{D}=\mu_{Y_{D}}$.

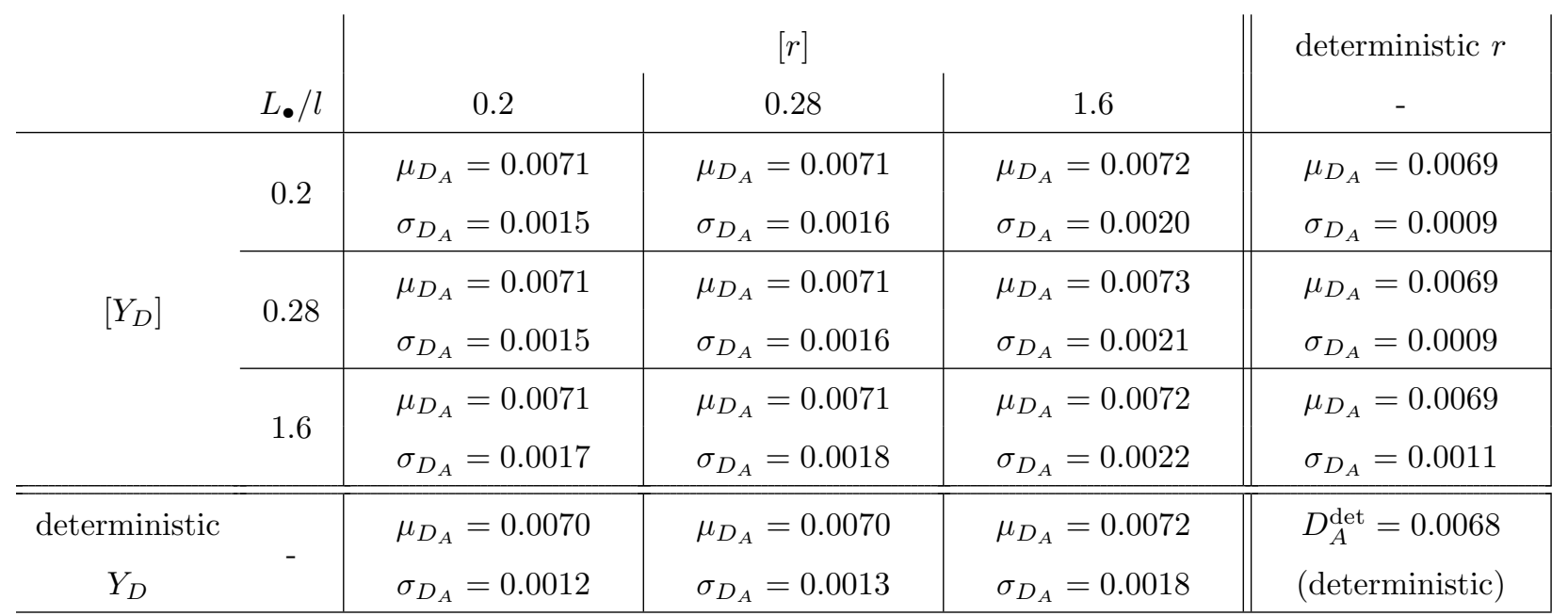

\subsubsection{Influence of the intermediate values on the global damage p-box}

The p-box resulting from all CDFs of each correlation length combination is depicted for the global damage $D_{A}$ in the top right of Figure 8 . To its left and bottom, the deviations $\Delta D_{A}=D_{A}-\hat{D}_{A}$ and $\Delta F_{D_{A}}=F_{D_{A}}-\hat{F}_{D_{A}}$ of each individual CDF to the corresponding midpoint line $\hat{\bullet}$ of the p-box are visualized, as defined in Figure 1. Those CDFs associated to a correlation length combination of the interval bounds are depicted in an individual color while those containing an intermediate correlation length are depicted in a gray dashed line. This way, the deviation plots clearly show, whether or not a simulation based on an intermediate correlation length falls outside the CDFs gained by the interval vertex. 

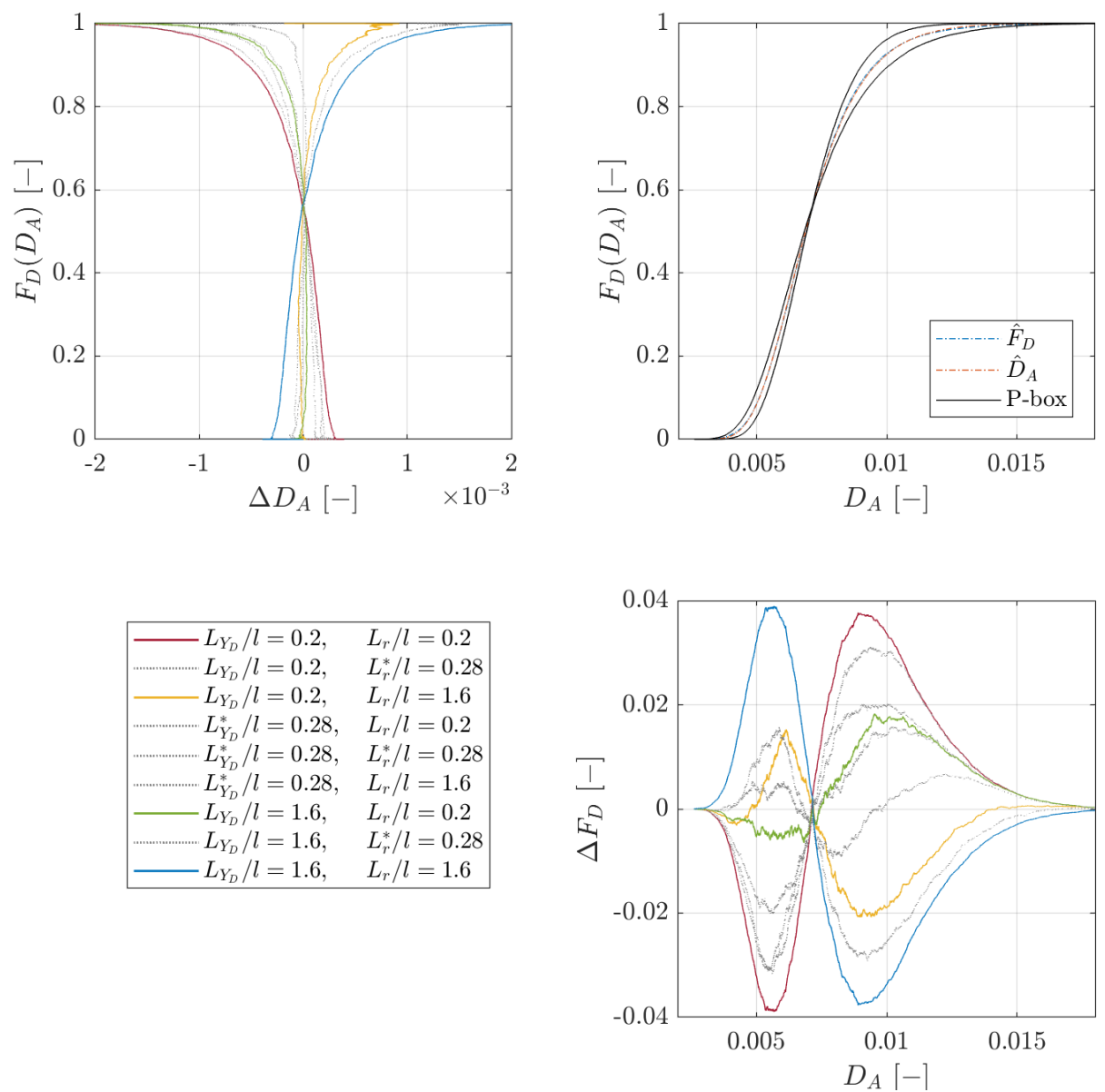

Figure 8: Resulting p-box (top right) of the global damage $D_{A}$ complemented by the deviation plots, $\Delta D_{A}$ (left) and $\Delta F_{D}$ (bottom), acquired with two imprecise random field input parameters, $[r]$ and $\left[Y_{D}\right]$.

As the different correlation lengths mainly affect the standard deviation but rarely the mean of the global damage, the p-box is very narrow around its mean and more pronounced towards its tails. As the latter are crucial within a reliability analysis, this underlines the importance of acknowledging epistemic uncertainties in the quantification of imprecise random fields. However, as can be seen from the deviation plots, the p-box of the global damage $D_{A}$ is affected by the interval vertex simulations only, the simulations including intermediate values can be neglected. Furthermore, the value of the global damage p-box is small as the main part of the beam remains undamaged. Still, the system can fail when the damage becomes too high locally. For this reason, the local damage $D$ is investigated in the following.

\subsubsection{Influence of the intermediate values on the local damage p-box}

Regarding the deviation plots of Figure 9 (point $A$ ) and Figure 10 (point $B$ ) the results of the local damage are much more noisy than those of the global damage. However, as the latter is an average value 

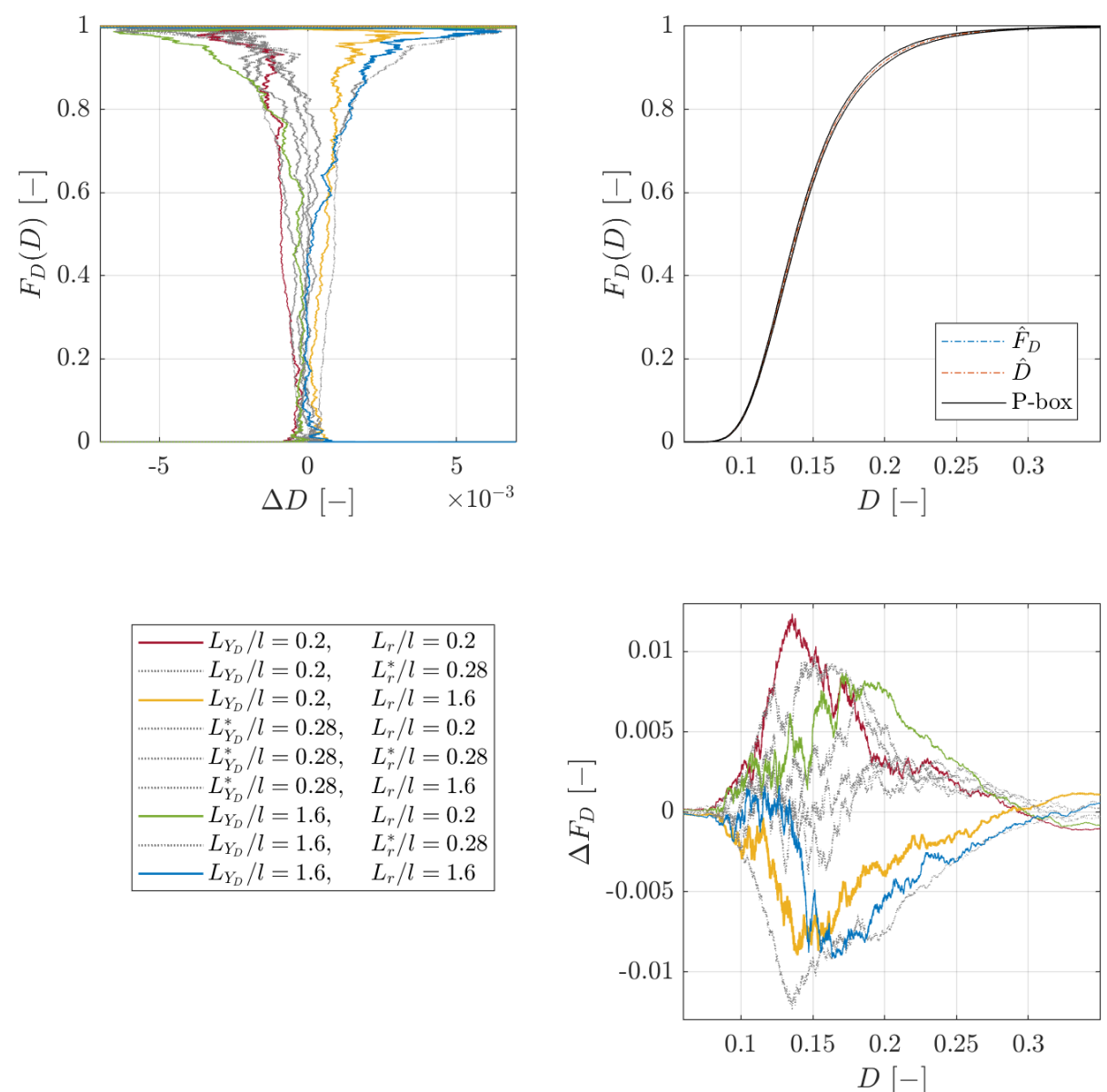

Figure 9: Resulting p-box (top right) of the local damage $D$ at point $A$ complemented by the deviation plots, $\Delta D$ (left) and $\Delta F_{D}$ (bottom), acquired with two imprecise random field input parameters, $[r]$ and $\left[Y_{D}\right]$.

of the whole structure it can be expected to be smoother than the local damage at a certain point entailing a higher variation. Furthermore, it can be seen that there are grey dashed lines outside the interval vertex results. This means that the p-box bounds are determined partly by intermediate values of the correlation length interval. However, compared to the range of $D$, the resulting p-boxes are very narrow. In the context of this problem, the correlation length seems not to affect the p-box of the local damage significantly from an engineering point of view. 

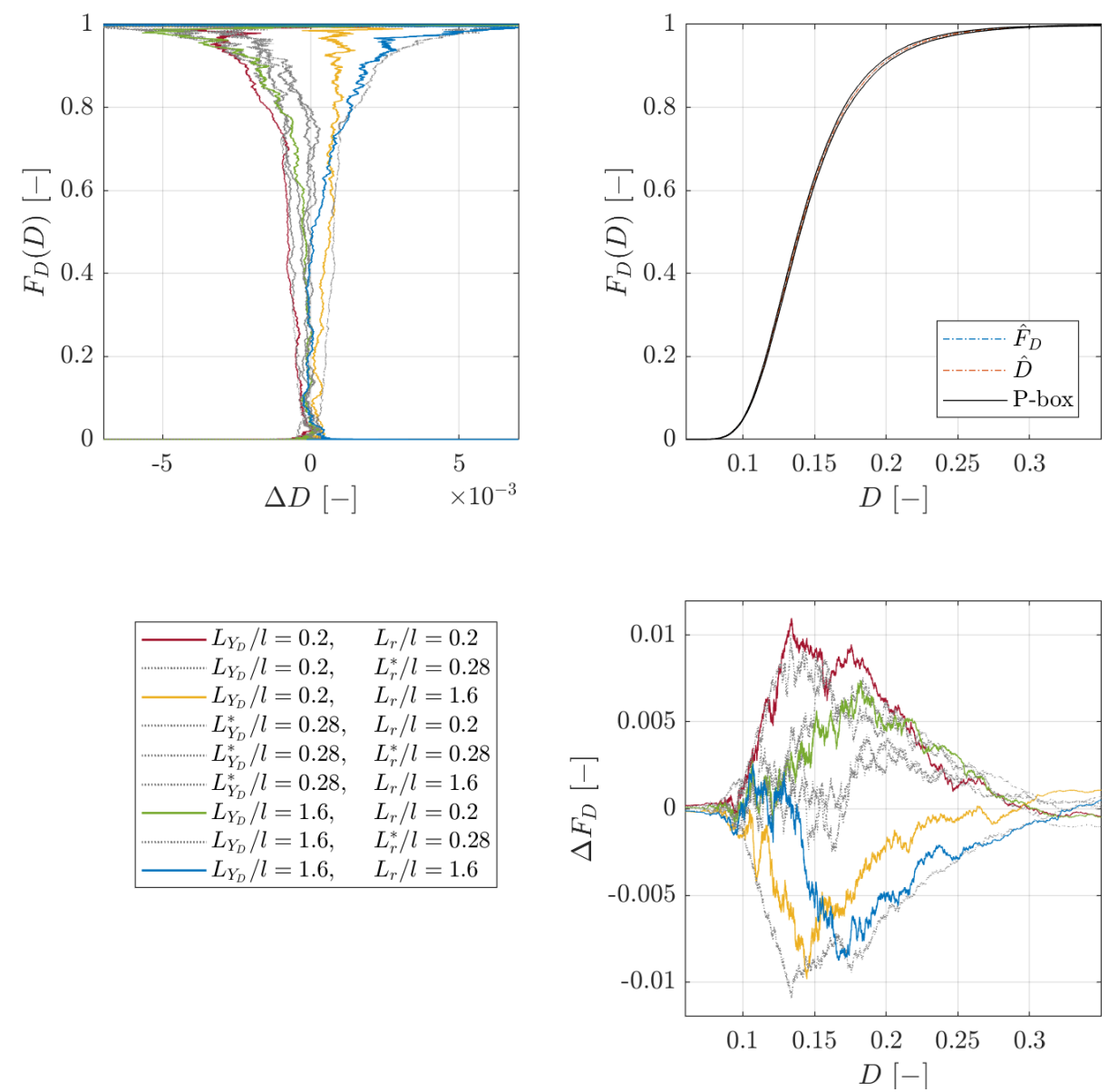

Figure 10: Resulting p-box (top right) of the local damage $D$ at point $B$ complemented by the deviation plots, $\Delta D$ (left) and $\Delta F_{D}$ (bottom), acquired with two imprecise random field input parameters, $[r]$ and $\left[Y_{D}\right]$. 


\subsubsection{Discussion of $S 1$}

From the engineering point of view, as the correlation length is hard to estimate, it is appropriate to assume a large epistemic uncertainty, as done in this example. It has been found that the intermediate value affects the p-box bounds at least in terms of the local damage. However, the following three issues remain to be investigated more carefully:

1. The intermediate value $L_{\bullet}^{*}=2.80 \mathrm{~m}$ is located close to the lower bounds of each interval valued correlation length $L_{\bullet}^{I}=[2,16] \mathrm{m}$. For this reason, it is not clear whether it affects the bounds only by its proximity. So this example is not sound enough to estimate the general meaning of intermediate values for interval valued correlation lengths independent of where they are located in the interval.

2. The interval bounds have the main impact on the p-box bounds. There are only small parts where the intermediate values determine the results. Those parts might be caused by an interference of the two imprecise random field inputs.

3. Comparing the results of the local damage with those of the global damage, it can be found that the latter are much smoother. This leads to the question, whether the local results are rather an artifact of the sampling than of the correlation length influence. The convergence of the mean value and standard deviation of the global and the local damage at point $A$ are depicted in Figure 11 and Figure 12 , respectively. Regarding the standard deviation, the global damage results of Figure $11 b$ indeed converge much faster than the standard deviation of the local damage given in Figure $12 \mathrm{~b}$.

To examine those three issues, a second simulation is investigated in the following section.

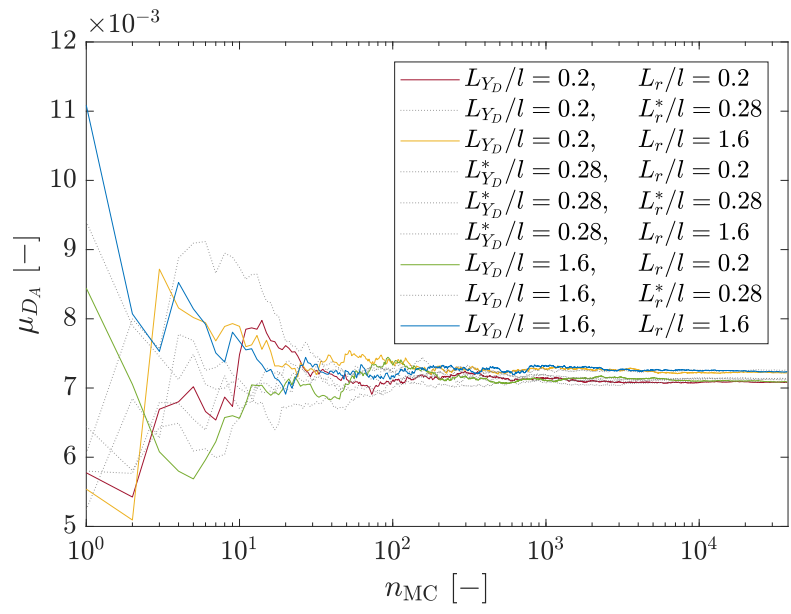

(a) Mean value $\mu_{D_{A}}$

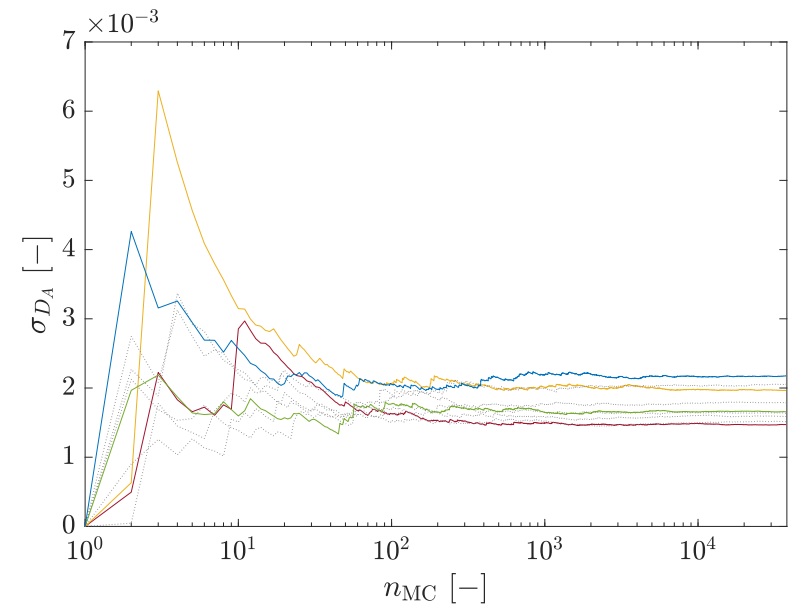

(b) Standard deviation $\sigma_{D_{A}}$

Figure 11: Mean value and the standard deviation of the global damage $D_{A}$ converging with increasing number of Monte Carlo samples $n_{\mathrm{MC}}$. 


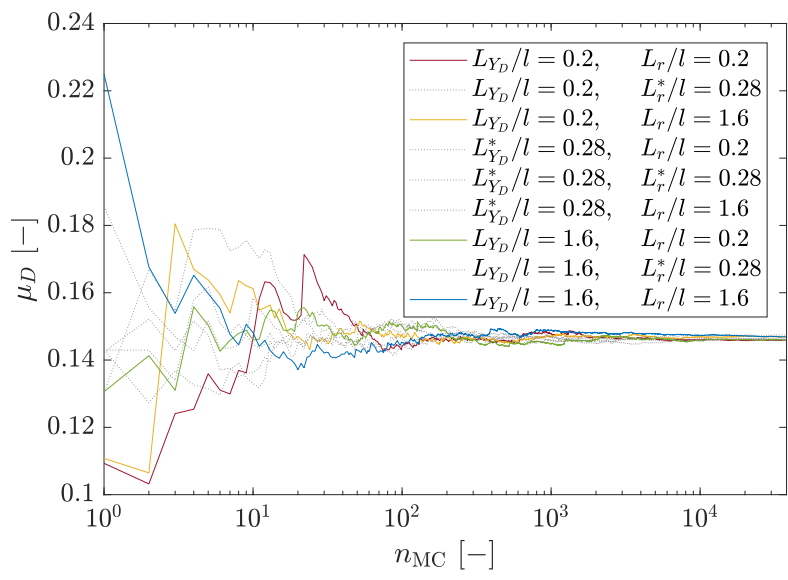

(a) Mean value $\mu_{D}$

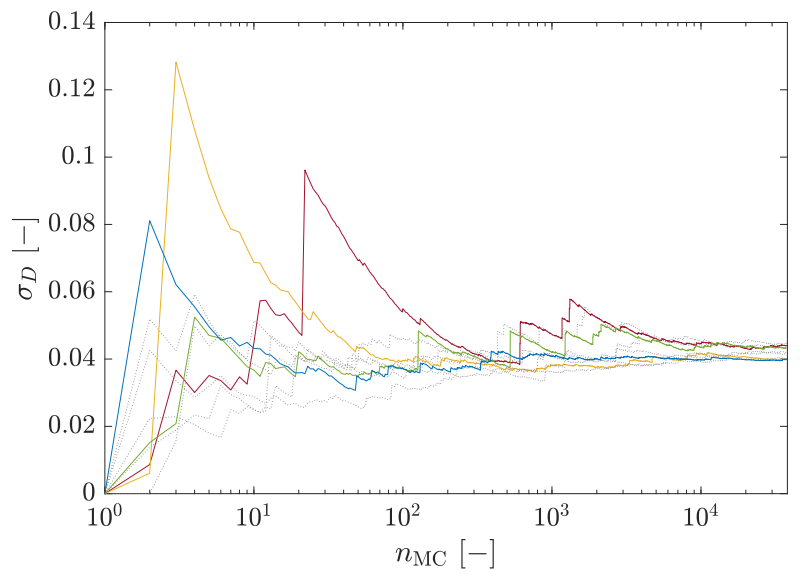

(b) Standard deviation $\sigma_{D}$

Figure 12: Mean value and the standard deviation of the local damage $D$ and point $A$ converging with increasing number of Monte Carlo samples $n_{\mathrm{MC}}$.

\subsection{Simulation 2: One imprecise random field using the same seed}

In this section, the effect of an interval valued correlation length that contains an intermediate value in its center is studied. For this purpose, the interval $L=[1,2] \mathrm{m}$ is chosen such that it encloses the intermediate value $L_{3}^{*}=1.48 \mathrm{~m}$. By using only a small interval, the influence on the variance resulting from different correlation lengths is much smaller. Furthermore, in order to exclude interfering effects of two imprecise random fields, $[r](\boldsymbol{z}, \theta)$ is considered as the only uncertain input parameter. According to Section 4.2 $r$ influences the quantity of interest much more than $Y_{D}$. To ensure that the results are not affected by convergence issues, the pseudo-random sampling of the standard normal distributed random variables describing the random field, see Eq. (24), is performed using the same seed. This means that the realizations of each correlation length simulation are created with the same values $\xi_{i}(\theta)$ than those of the other correlation lengths. The simulation is performed using Monte Carlo simulation with a set of $n_{\mathrm{MC}}=20000$ samples.

\subsubsection{Influence of the central intermediate value on the global damage p-box}

The p-box and its corresponding deviation plots of the global damage $D_{A}$ are depicted in Figure 13 . The effect of the interval valued correlation length on the p-box is qualitatively the same as before. The width $\Delta D_{A}$ of the p-box is much smaller which can be traced back to the smaller correlation length interval.

The CDF of the intermediate value is located centred within the p-box. This can be concluded from the deviation plots, where the gray dashed line runs close to zero compared to the ones corresponding to the interval bounds. This correlates to the fact that the intermediate correlation length is situated centered within the correlation length interval. Furthermore, around the mean value, the intermediate correlation 

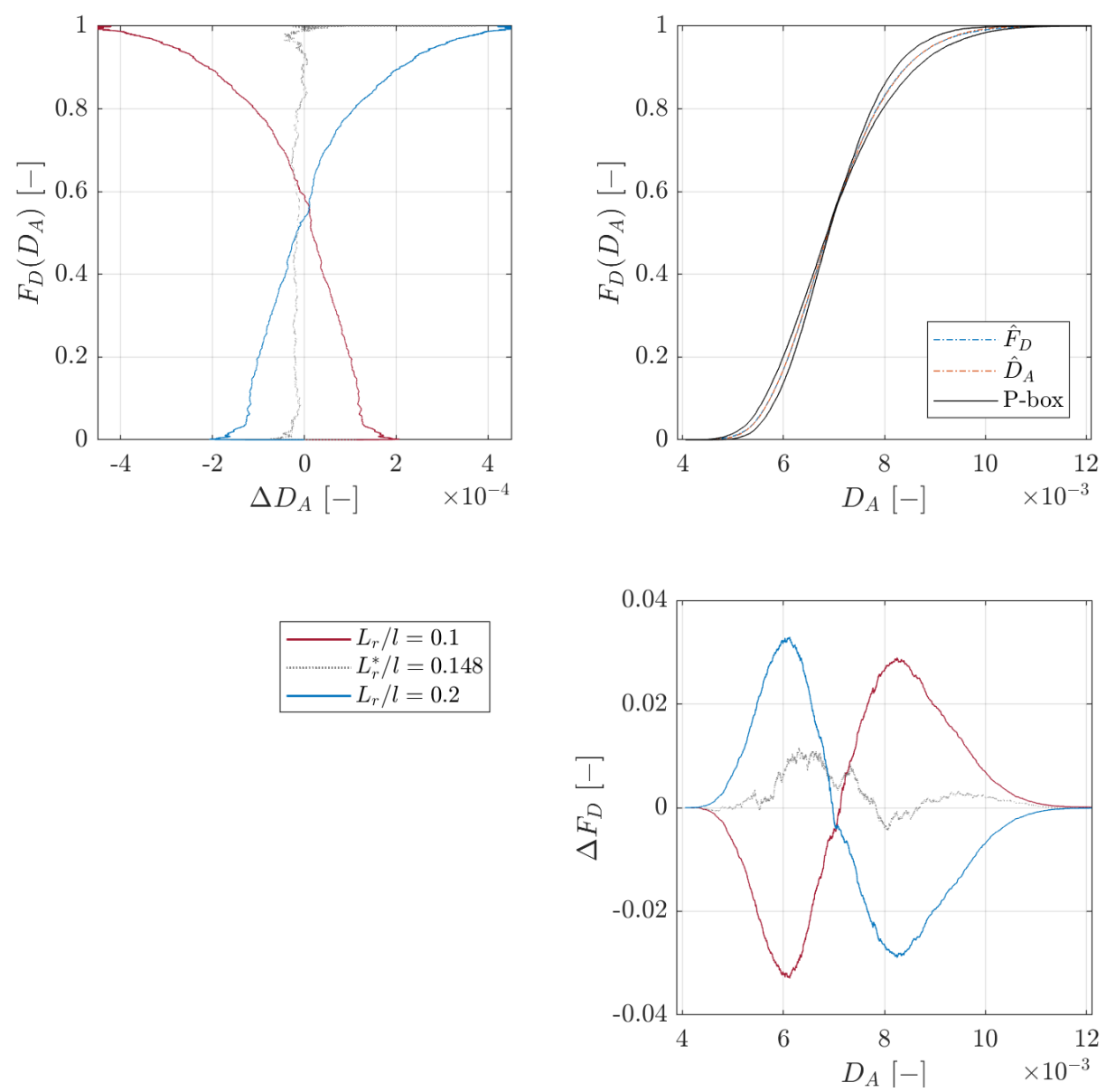

Figure 13: Resulting p-box (top right) of the global damage $D_{A}$ complemented by the deviation plots, $\Delta D_{A}$ (left) and $\Delta F_{D}$ (bottom), acquired with one imprecise random field input parameter $[r]$.

length falls outside the interval bounds. Still, as the tails are more interesting in terms of reliability analysis, from the engineering point of view this can be neglected.

\subsubsection{Influence of the central intermediate value on the local damage p-box}

Regarding the local damage $D$ at the points $A$ and $B$ in Figure 14 and Figure 15 , respectively, the p-box is even less pronounced than in S1. As the interval of the correlation length is much smaller than within the first simulation, this could be expected. However, although the intermediate value is in the center of the interval, the corresponding CDF varies significantly from the center of the p-box. It even falls outside the interval results at several points what means that the p-box is - strictly spoken - depending on the intermediate value. Although the p-box of the local damage is not very pronounced and therefore negligible from an engineering point of view, the dependency on intermediate values can be shown theoretically. It 
cannot be ruled out that intermediate values have a much more important impact regarding other uncertain input parameters, quantities of interest or application fields.
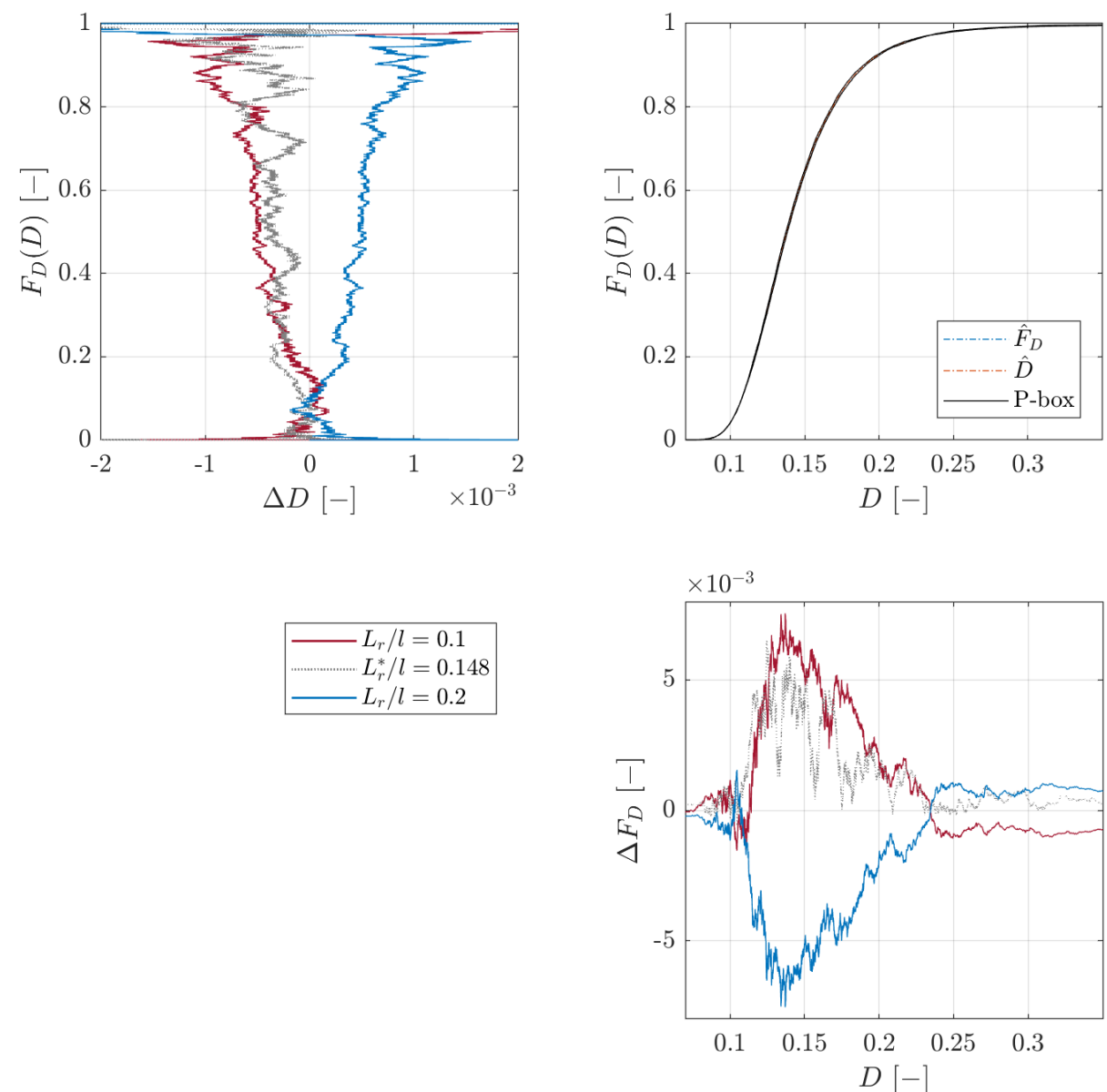

Figure 14: Resulting p-box (top right) of the local damage $D$ at point $A$ complemented by the deviation plots, $\Delta D$ (left) and $\Delta F_{D}$ (bottom), acquired with one imprecise random field input parameter $[r]$.

\subsubsection{Discussion of S2}

To investigate the open issues of Section 5.1, a second example has been constructed in this section. Only one imprecise random field $[r]$ has been considered. The interval valued correlation length has been regarded much smaller to preempt significant differences in the local variance. Furthermore, the interval has been chosen such that it encloses an intermediate value in its center. For each correlation length simulation, the realizations of the random field have been determined using the same random variables.

The simulation S2 supports the findings of the first simulation. Although the intermediate value is situated in the center of the interval valued correlation length, it can be shown that it determines - theoretically - the p-box bounds. As only one imprecise random field has been used it can be excluded that this is due 

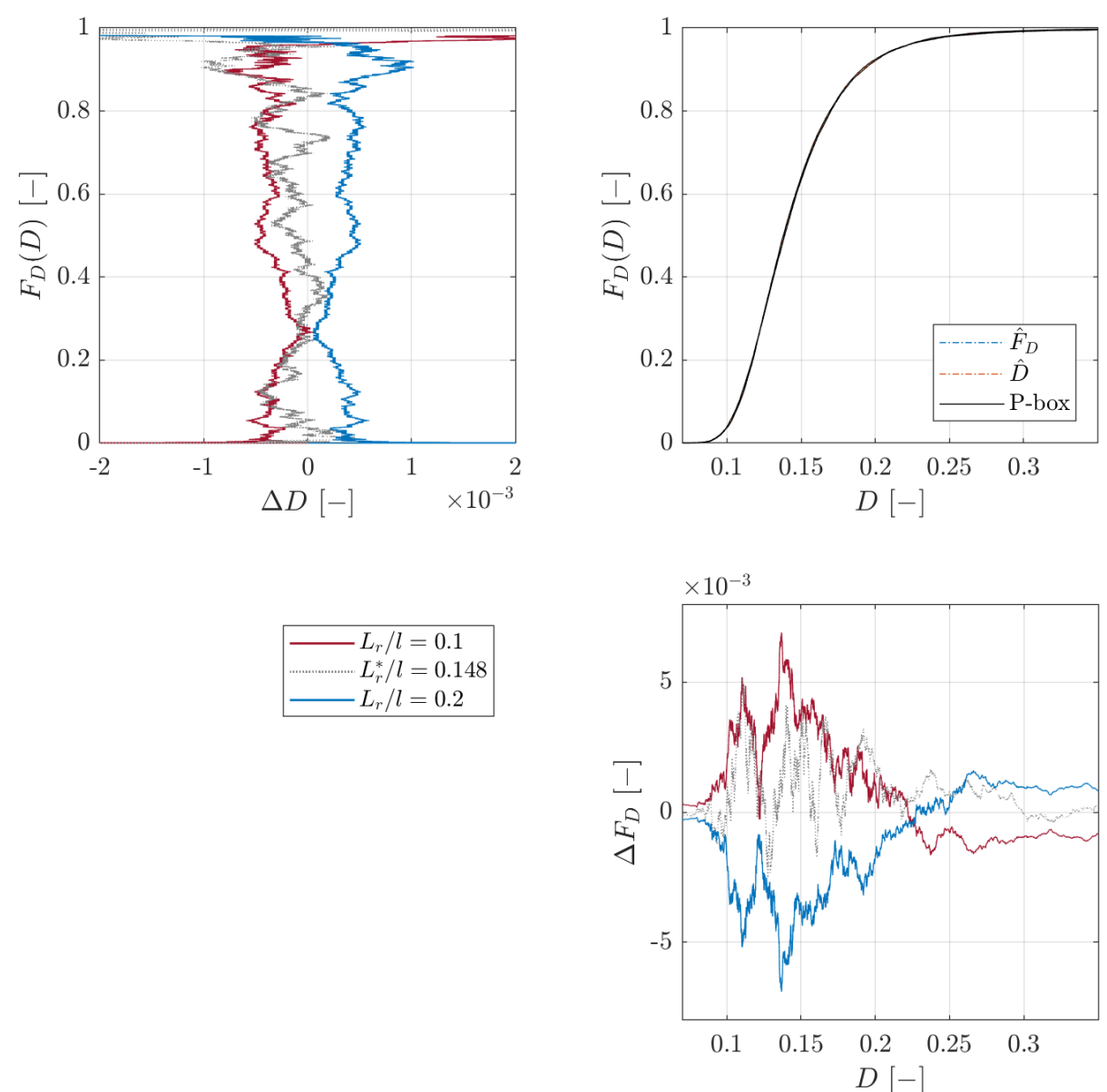

Figure 15: Resulting p-box (top right) of the local damage $D$ at point $B$ complemented by the deviation plots, $\Delta D$ (left) and $\Delta F_{D}$ (bottom), acquired with one imprecise random field input parameter $[r]$.

to an interference of several fields. As the sampling has been performed using the same seed, it can further be assured that this not due to sampling or convergence issues.

Still, as the p-box of the local damage is not pronounced, the influence of intermediate values to the p-box bounds can only be shown theoretically. From an engineering point of view, the effort of a simulation including imprecise random fields can only be justified if the global damage is of interest. In this case, a pure vertex analysis has been shown to be sufficient. It should be noted that intermediate values are expected to be much more significant for non-monotonic problems.

\section{Conclusion}

Spatial properties containing both aleatory and epistemic uncertain aspects can be modelled by imprecise random fields. Considering such input parameters in a finite element analysis means that the quantity of interest is described by a probability box (p-box). Regarding interval valued correlation lengths, intermediate 
values can become crucial for the p-box bounds due to a possible non-monotonic relation between the correlation length and the bounds on the responses of the model. The intermediate values can be found a-priori by an optimization algorithm.

In this paper, the influence of one and two imprecise random field inputs within a non-linear finite element model has been examined for a brittle damage problem. For this purpose, the damage evolution of a concrete four-point bending beam has been simulated in a p-box framework using Monte Carlo simulation in combination with a vertex analysis on the epistemic uncertainty. As quantities of interest, the global damage of the beam as well as the local damage at two points of the beam have been considered.

It could be shown that an interval valued correlation length can highly affect the p-box of the quantity of interest, especially by influencing the standard deviation. As the correlation length is often difficult to determine, acknowledging this lack of knowledge can be crucial for the sensitive but important tails within a reliability analysis.

Furthermore, the importance of the intermediate correlation lengths to the p-box bounds has been investigated. By the results presented in this paper it could be shown that, speaking from an engineering point of view, for this application case the bounds of the p-box are defined by the bounds of the interval. This way, the computational cost to propagate imprecise random fields can be reduced significantly by performing only a vertex analysis of the interval bounds. Technically speaking, some bounds of the p-box are defined by the intermediate values (e.g., the local damage in points A and B in simulation S1). However, in this case the effect could be shown to be negligible, and hence, the additional computational cost of (i) finding the intermediate values and (ii) propagating the corresponding random fields, is not justified. Globally, the change of the correlation length changes the variance of the mean, which causes the change of the CDFs depicted in Figure 7. Therefore, these two arguments could explain why little influence of the intermediate correlation lengths was observed. For an other problem, intermediate values could be much more relevant than the present problem, but this is a topic for future research. Of course, one should keep in mind that the intermediate values only provide bounds in case strict monotonicity with respect to the sample path realizations is maintained within the analysis. This is for instance generally not true in structural dynamics.

\section{Acknowledgements}

The support of the German Research Foundation (DFG) during the priority program SPP 1886 (NA330/121) is gratefully acknowledged. Matthias Faes acknowledges the support of the Research Foundation Flanders (FWO) under grant number 12P3519N. The support of the French-German University is acknowledged under the French-German doctoral college "Sophisticated Numerical and Testing Approaches (SNTA), grant 
Hannover, the Lower Saxony Ministry of Science and Culture (MWK) and the German Research Foundation (DFG).

\section{References}

[1] A. D. Kiureghian, O. Ditlevsen, Aleatory or epistemic? does it matter?, Structural Safety 31 (2) (2009) 105 - 112, risk Acceptance and Risk Communication. doi:https://doi.org/10.1016/j.strusafe.2008.06.020

[2] R. Moore, R. Kearfott, M. Cloud, Introduction to interval analysis, Society for Industrial and applied Mathematics, 2009.

[3] M. Hanss, Applied fuzzy arithmetic: an introduction with engineering applications, Springer, 2005.

[4] M. Beer, S. Ferson, V. Kreinovich, Imprecise probabilities in engineering analyses, Mechanical Systems and Signal Processing 37 (1) (2013) 4-29. doi:http://dx.doi.org/10.1016/j.ymssp.2013.01.024

[5] M. Eldred, L. Swiler, G. Tang, Mixed aleatory-epistemic uncertainty quantification with stochastic expansions and optimization-based interval estimation, Reliability Engineering \& System Safety 96 (9) (2011) 1092 - 1113, quantification of Margins and Uncertainties. doi:https://doi.org/10.1016/j.ress.2010.11.010

[6] M. Götz, W. Graf, M. Kaliske, Structural design with polymorphic uncertainty models, International Journal of Reliability and Safety $9(2 / 3)(2015) 112-131$.

[7] M. Fina, P. Weber, W. Wagner, Polymorphic uncertainty modeling for the simulation of geometric imperfections in probabilistic design of cylindrical shells, Structural Safety 82 (October 2019) (2020) 101894. doi:10.1016/j.strusafe. 2019.101894

[8] J. C. Helton, J. D. Johnson, W. L. Oberkampf, An exploration of alternative approaches to the representation of uncertainty in model predictions, Reliability Engineering and System Safety 85 (1-3) (2004) 39-71. doi:10.1016/j.ress.2004.03.025

[9] B. Möller, B. Michael, Fuzzy Randomness: Uncertainty in Civil Engineering and Computational Mechanics, 2004.

[10] S. Ferson, V. Kreinovich, L. Grinzburg, D. Myers, K. Sentz, Constructing probability boxes and dempster-shafer structures, Sandia journal manuscript; Not yet accepted for publication.

[11] C. Wang, H. Zhang, M. Beer, Computing tight bounds of structural reliability under imprecise probabilistic information, Computers and Structures 208 (2018) 92-104. doi:10.1016/j.compstruc.2018.07.003

[12] P. Wei, J. Song, S. Bi, M. Broggi, M. Beer, Z. Lu, Z. Yue, Non-intrusive stochastic analysis with parameterized imprecise probability models: I. Performance estimation, Mechanical Systems and Signal Processing 124 (2019) 349-368.

[13] J. Song, M. Valdebenito, P. Wei, M. Beer, Z. Lu, Non-intrusive imprecise stochastic simulation by line sampling, Structural Safety 84 (2020) 101936.

[14] A. Sofi, G. Muscolino, F. Giunta, Propagation of uncertain structural properties described by imprecise Probability Density Functions via response surface method, Probabilistic Engineering Mechanics 60. doi:10.1016/j.probengmech. 2020.103020

[15] R. Schöbi, B. Sudret, Global sensitivity analysis in the context of imprecise probabilities (p-boxes) using sparse polynomial

chaos expansions, Reliability Engineering \& System Safety 187 (2019) 129-141. doi:https://doi.org/10.1016/j.ress. 2018.11.021

[16] J. Sadeghi, M. de Angelis, E. Patelli, Robust propagation of probability boxes by interval predictor models, Structural Safety 82 (2020) 101889. doi:https://doi.org/10.1016/j.strusafe.2019.101889.

[17] B. Sudret, A. Der Kiureghian, Stochastic finite element methods and reliability - a state-of-the-art report, Tech. rep., Department of Civil \& Environmental Engineering, University of California, Berkeley, report No. UCB/SEMM-2000/08 (2000).

[18] D. C. Charmpis, G. I. Schuëller, M. F. Pellissetti, The need for linking micromechanics of materials with stochastic finite elements: a challenge for materials science, Computational Materials Science 41 (1) (2007) 27-37. 
[19] D. M. Do, W. Gao, C. Song, Stochastic finite element analysis of structures in the presence of multiple imprecise random field parameters, Computer Methods in Applied Mechanics and Engineering 300 (2016) 657 - 688.

[20] F. N. Schietzold, A. Schmidt, M. M. Dannert, A. Fau, R. M. Fleury, W. Graf, M. Kaliske, C. Könke, T. Lahmer, U. Nackenhorst, Development of fuzzy probability based random fields for the numerical structural design, GAMM Mitteilungen 42 (1) (2019) 1-19. doi:10.1002/gamm.201900004

[21] M. Faes, M. A. Valdebenito, D. Moens, M. Beer, Bounding the first excursion probability of linear structures subjected to imprecise stochastic loading, Computers and Structures (2020) 106320.

[22] M. M. Dannert, R. M. Fleury, A. Fau, U. Nackenhorst, Non-linear finite element analysis under mixed epistemic and aleatory uncertain random field input, in: M. Beer, E. Zio (Eds.), Proceedings of the 29th European Safety and Reliability Conference, 2019, pp. 2693-2698.

[23] M. Faes, D. Moens, Imprecise random field analysis with parametrized kernel functions, Mechanical Systems and Signal Processing 134 (2019) 106334. doi:10.1016/j.ymssp.2019.106334

[24] W. Betz, I. Papaioannou, D. Straub, Numerical methods for the discretization of random fields by means of the KarhunenLoève expansion, Computer Methods in Applied Mechanics and Engineering 271 (2014) 109-129.

[25] S. Montoya-Noguera, T. Zhao, Y. Hu, Y. Wang, K.-K. Phoon, Simulation of non-stationary non-Gaussian random fields from sparse measurements using Bayesian compressive sampling and Karhunen-Loève expansion, Structural Safety 79 (September 2018) (2019) 66-79. doi:10.1016/j.strusafe.2019.03.006

[26] M. Faes, D. Moens, Recent trends in the modeling and quantification of non-probabilistic uncertainty, Archives of Computational Methods in Engineeringdoi:10.1007/s11831-019-09327-x

[27] M. Beer, Y. Zhang, S. T. Quek, K. K. Phoon, Reliability analysis with scarce information: Comparing alternative approaches in a geotechnical engineering context, Structural Safety 41 (2013) 1-10. doi:10.1016/j.strusafe.2012.10.003

[28] M. Faes, G. D. Sabyasachi, D. Moens, Hybrid spatial uncertainty analysis for the estimation of imprecise failure probabilities in Laser Sintered PA-12 parts, Computers \& Mathematics with Applications 78 (7) (2019) 2395-2406. doi:https://doi.org/10.1016/j.camwa.2018.08.056

[29] M. Beer, V. Kreinovich, Interval or moments: Which carry more information?, Soft Computing 17 (8) (2013) $1319-1327$. doi:10.1007/s00500-013-1002-1

[30] E. Vanmarcke, Random Fields: Analysis and Synthesis, MIT Press, Cambridge, 1983.

[31] P. Spanos, R. Ghanem, Stochastic finite element expansion for random media, Journal of engineering mechanics 115 (5) (1989) 1035-1053.

[32] M. Beer, I. A. Kougioumtzoglou, E. Patelli, Emerging Concepts and Approaches for Efficient and Realistic Uncertainty Quantification, in: Maintenance and Safety of Aging Infrastructure, Structures \& Infrastructures Series, CRC Press, 2014, pp. 121-161. doi:doi:10.1201/b17073-6

[33] R. G. Ghanem, P. D. Spanos, Stochastic Finite Elements: A Spectral Approach, Springer-Verlag New York, Inc., New York, NY, USA, 1991.

[34] S. P. Huang, S. T. Quek, K. K. Phoon, Convergence study of the truncated karhunenloeve expansion for simulation of stochastic processes, International Journal for Numerical Methods in Engineering 52 (9) (2001) 1029-1043. doi: $10.1002 / \mathrm{nme} .255$

[35] J. Ching, K.-K. Phoon, Impact of Autocorrelation Function Model on the Probability of Failure, Journal of Engineering Mechanics 145 (1) (2019) 04018123. doi:10.1061/(asce)em.1943-7889.0001549

[36] W. Verhaeghe, W. Desmet, D. Vandepitte, D. Moens, Interval fields to represent uncertainty on the output side of a static FE analysis, Computer Methods in Applied Mechanics and Engineering 260 (2013) 50-62. doi:10.1016/j.cma.2013.03. 021 . 
[37] M. Faes, D. Moens, On auto and crossinterdependence in interval field finite element analysis, International Journal for Numerical Methods in Engineering 121 (9) (2020) 2033-2050. doi:10.1002/nme.6297.

[38] M. Faes, D. Moens, Identification and quantification of spatial interval uncertainty in numerical models, Computers and Structures 192 (2017) 16-33. doi:10.1016/j.compstruc.2017.07.006

[39] M. M. Dannert, A. Fau, R. M. Fleury, M. Broggi, U. Nackenhorst, M. Beer, A probability-box approach on uncertain correlation lengths by stochastic finite element method, PAMM 18 (1) (2018) e201800114. doi:10.1002/pamm.201800114

[40] W. Gao, D. Wu, K. Gao, X. Chen, F. Tin-loi, Structural reliability analysis with imprecise random and interval fields, Applied mathematical modelling 55 (2018) 49-67. doi:10.1016/j.apm.2017.10.029

[41] S.-K. Au, J. L. Beck, Estimation of small failure probabilities in high dimensions by subset simulation, Probabilistic Engineering Mechanics 16 (4) (2001) 263-277. doi:https://doi.org/10.1016/S0266-8920(01)00019-4

[42] H. J. Pradlwarter, G. I. Schuëller, P. S. Koutsourelakis, D. C. Charmpis, Application of line sampling simulation method to reliability benchmark problems, Structural Safety 29 (3) (2007) 208-221.

[43] M. A. Misraji, M. A. Valdebenito, H. A. Jensen, C. F. Mayorga, Application of directional importance sampling for estimation of first excursion probabilities of linear structural systems subject to stochastic Gaussian loading, Mechanical Systems and Signal Processing 139 (2020) 106621.

[44] J. Lemaitre, R. Desmorat, Engineering damage mechanics: ductile, creep, fatigue and brittle failures, Springer Science \& Business Media, 2005.

[45] R. Desmorat, S. Cantournet, Modeling microdefects closure effect with isotropic/anisotropic damage, International Journal of Damage Mechanics 17 (1) (2008) 65-96.

[46] M. Fassin, S. Wulfinghoff, S. Reese, A gradient-extended elastic isotropic damage model considering crack-closure, in: Proceedings of the 7th GACM Colloquium on Computational Mechanics for Young Scientists from Academia and Industry, 2017, pp. 305-311.

[47] J. Marigo, Formulation d'une loi d'endommagement d'un matériau élastique, CR Acad. Sci. Paris 292 (1981) $1309-1312$. 